\title{
Three-dimensional tricritical spins and polymers
}

\author{
Roland Bauerschmidt, Martin Lohmann ${ }^{\dagger}$ and Gordon Slade ${ }^{\dagger}$
}

\begin{abstract}
We consider two intimately related statistical mechanical problems on $\mathbb{Z}^{3}$ : (i) the tricritical behaviour of a model of classical unbounded $n$-component continuous spins with a triple-well single-spin potential (the $|\varphi|^{6}$ model), and (ii) a random walk model of linear polymers with a three-body repulsion and two-body attraction at the tricritical theta point (critical point for the collapse transition) where repulsion and attraction effectively cancel. The polymer model is exactly equivalent to a supersymmetric spin model which corresponds to the $n=0$ version of the $|\varphi|^{6}$ model. For the spin and polymer models, we identify the tricritical point, and prove that the tricritical two-point function has Gaussian long-distance decay, namely $|x|^{-1}$. The proof is based on an extension of a rigorous renormalisation group method that has been applied previously to analyse the $|\varphi|^{4}$ and weakly self-avoiding walk models on $\mathbb{Z}^{4}$.
\end{abstract}

Keywords: self-avoiding walk, spin system, tricritical point, polymer collapse, renormalisation group, supersymmetry.

MSC2010 Classifications: Primary 82B27, 82B28 82B41; Secondary 60K35.

\section{Introduction and main results}

In statistical mechanics, it often occurs that variation of a parameter leads abruptly to passage from one phase to another as the parameter passes through a critical value. Prominent examples are freezing, evaporation, superconductivity, Bose-Einstein condensation, or the metal-insulator transition. The mathematically best understood examples include the Ising model and percolation. In many cases, the critical point separates an ordered (low-temperature) phase from a disordered (high-temperature) phase. The universal behaviour at and near the critical point is a phenomenon of great interest.

In this paper, we construct the tricritical point of the $n$-component $|\varphi|^{6}$ model $(n \geq 1)$ on the 3-dimensional cubic lattice $\mathbb{Z}^{3}$. The tricritical point is conjecturally a point of confluence of lines of first-order and second-order phase transition. We also analyse a polymer model on $\mathbb{Z}^{3}$ having a three-body repulsion and two-body attraction, with parameters adjusted to be at the tricritical theta point where repulsion and attraction effectively cancel. For the polymer model, the tricritical

*Department of Pure Mathematics and Mathematical Statistics, University of Cambridge, Centre for Mathematical Sciences, Wilberforce Road, Cambridge, CB3 0WB, UK. https://orcid.org/0000-0001-7453-2737. E-mail: rb812@cam.ac.uk

${ }^{\dagger}$ Department of Mathematics, University of British Columbia, Vancouver, BC, Canada V6T 122. Lohmann: https://orcid.org/0000-0002-6627-638X. Slade: https://orcid.org/0000-0001-9389-9497. E-mail: marlohmann@math.ubc.ca, slade@math.ubc.ca 
point conjecturally divides a curve of critical points into an arc of self-avoiding walk critical points and an arc of critical points for polymer collapse. In each case, we prove Gaussian decay of the tricritical two-point function, i.e. $|x|^{-1}$ decay.

Tricritical behaviour has been much less studied mathematically than critical behaviour. One reason is that techniques effective in the analysis of the critical behaviour, such as correlation inequalities, cannot be used to identify a multicritical point. An exception to this is the renormalisation group (RG) approach. Our proof is based on the rigorous RG method of [16], which has been applied previously to analyse the critical behaviour of the $n$-component $|\varphi|^{4}$ model and of the weakly self-avoiding walk, on the 4 -dimensional integer lattice $\mathbb{Z}^{4}$ (see, e.g., $[3,5,8]$ ), as well as for long-range models below the upper critical dimension [30,34]. A substantial part of previous papers in this RG scheme - and the main one that depends on the specific model - is taken up by perturbation theory. One of the contributions of our work is to simplify and shorten the treatment of perturbation theory, and a principal focus in the paper is on this aspect.

The polymer model we study is exactly equivalent to a supersymmetric spin model which corresponds to the $n=0$ version of the $|\varphi|^{6}$ model, and our treatment for $n \geq 1$ and $n=0$ is unified. The fact that the polymer theta point should be investigated as the $n=0$ version of the spin problem was clarified in the physics literature in the 1980s $[17,18]$. Although the tricritical theory of $|\varphi|^{6}$ spins is a standard part of the physical theory, a mathematical treatment has been lacking, apart from initial steps taken for a hierarchical model in [32]. For the polymer problem, there are no previous mathematical results on the theta point for $\mathbb{Z}^{d}$; the combination of repulsion and attraction makes the polymer model even more difficult than the self-avoiding walk, which is purely repulsive.

We begin with precise definitions of the two models and a precise statement of our results.

\section{$1.1|\varphi|^{6}$ model}

Fix $L>1$ and let $\Lambda=\Lambda_{N}$ denote the 3-dimensional discrete torus of period $L^{N}$. We are interested in the infinite-volume limit $N \rightarrow \infty$. We write $\Delta$ for the Laplace operator on functions $f: \Lambda \rightarrow \mathbb{R}$, defined by

$$
(\Delta f)_{x}=\sum_{y \in \Lambda:|y-x|_{1}=1}\left(f_{y}-f_{x}\right) .
$$

We use the same symbol $\Delta$ for the Laplacian on $\mathbb{Z}^{3}$; the meaning should be clear from context. The Laplacian operates component-wise on vector-valued functions. The lattice Green function $C_{0, x}$ is the matrix element of the inverse of $-\Delta_{\mathbb{Z}^{3}}$, and its diagonal element $C_{0,0}$ plays a role in our results.

Let $n \geq 1$ be an integer. The spin field is a function $\varphi: \Lambda \rightarrow \mathbb{R}^{n}$. Given $a>0$ and $g, \nu \in \mathbb{R}$, let

$$
V\left(\varphi_{x}\right)=\frac{1}{8} a\left|\varphi_{x}\right|^{6}+\frac{1}{4} g\left|\varphi_{x}\right|^{4}+\frac{1}{2} \nu\left|\varphi_{x}\right|^{2}
$$

where $\left|\varphi_{x}\right|^{2}=\sum_{i=1}^{n}\left(\varphi_{x}^{i}\right)^{2}$. The partition function is

$$
Z_{a, g, \nu ; n, N}=\int_{\left(\mathbb{R}^{n}\right)^{\Lambda}} e^{-\sum_{x \in \Lambda}\left(V\left(\varphi_{x}\right)+\frac{1}{2} \varphi_{x} \cdot\left(-\Delta \varphi_{x}\right)\right)} \prod_{x \in \Lambda} d \varphi_{x}
$$


and the expectation of a function $F$ of the spin field is written as

$$
\langle F\rangle_{a, g, \nu ; n, N}=\frac{1}{Z_{a, g, \nu ; n, N}} \int_{\left(\mathbb{R}^{n}\right)^{\Lambda}} F(\varphi) e^{-\sum_{x \in \Lambda}\left(V\left(\varphi_{x}\right)+\frac{1}{2} \varphi_{x} \cdot\left(-\Delta \varphi_{x}\right)\right)} \prod_{x \in \Lambda} d \varphi_{x}
$$

The finite-volume two-point function and susceptibility are defined respectively by

$$
\begin{aligned}
G_{N ; 0, x}(a, g, \nu ; n) & =\frac{1}{n}\left\langle\varphi_{0} \cdot \varphi_{x}\right\rangle_{a, g, \nu ; n, N} . \\
\chi_{N}(a, g, \nu ; n) & =\frac{1}{n} \sum_{x \in \Lambda}\left\langle\varphi_{0} \cdot \varphi_{x}\right\rangle_{a, g, \nu ; n, N} .
\end{aligned}
$$

Their $N \rightarrow \infty$ limits (assuming they exist) are denoted $G_{0, x}(a, g, \nu ; n)$ and $\chi(a, g, \nu ; n)$.

\subsection{Polymer model}

The polymer model is defined in terms of $X=(X(t))_{t \geq 0}$, which denotes the continuous-time simple random walk on the discrete torus $\Lambda_{N}$ with nearest-neighbour steps occurring at the events of a rate-6 Poisson process (here "6" is the degree of $\Lambda_{N}$ ). We write $E_{N}$ for the expectation when $X(0)=0$.

For $x \in \Lambda$ and $T \geq 0$, the random variable

$$
L_{T, x}=\int_{0}^{T} \mathbb{1}_{X(t)=x} d t
$$

denotes the local time at $x$ up to time $T$. For fixed $a>0$, and for $g \in \mathbb{R}$ and $x \in \Lambda_{N}$, we define

$$
\begin{aligned}
c_{T, N}(a, g ; x) & =E_{N}\left(e^{-\sum_{y \in \Lambda_{N}}\left(a L_{T, y}^{3}+g L_{T, y}^{2}\right)} \mathbb{1}_{X(T)=x}\right), \\
c_{T, N}(a, g) & =\sum_{x \in \Lambda_{N}} c_{T, N}(a, g ; x)=E_{N}\left(e^{-\sum_{x \in \Lambda_{N}}\left(a L_{T, x}^{3}+g L_{T, x}^{2}\right)}\right) .
\end{aligned}
$$

Note that, by definition,

$$
\sum_{x \in \Lambda_{N}} L_{T, x}^{2}=\int_{0}^{T} \int_{0}^{T} \mathbb{1}_{X(s)=X(t)} d s d t, \quad \sum_{x \in \Lambda_{N}} L_{T, x}^{3}=\int_{0}^{T} \int_{0}^{T} \int_{0}^{T} \mathbb{1}_{X(s)=X(t)=X(u)} d s d t d u .
$$

We are interested in $g<0$; in this case there is an attractive two-body term and a competing repulsive three-body term in the exponent on the right-hand side of (1.8).

The finite-volume two-point function and susceptibility are defined respectively by

$$
G_{N ; 0, x}(a, g, \nu ; 0)=\int_{0}^{\infty} c_{T}(a, g ; x) e^{-\nu T} d T, \quad \chi_{N}(a, g, \nu ; 0)=\int_{0}^{\infty} c_{T}(a, g) e^{-\nu T} d T .
$$

The finite-volume susceptibility is finite for all $(g, \nu) \in \mathbb{R}^{2}$ (and hence so is the two-point function), since by Hölder's inequality $T=\sum_{x \in \Lambda} L_{T, x} \leq\left(\sum_{x \in \Lambda} L_{T, x}^{3}\right)^{1 / 3}|\Lambda|^{2 / 3}$, and also $\sum_{x \in \Lambda} L_{T, x}^{2} \leq$ $\left(\sup _{x} L_{T, x}\right) \sum_{x} L_{T, x} \leq T^{2}$, so

$$
c_{T, N}(a, g) e^{-\nu T} \leq e^{-a T^{3}|\Lambda|^{-2}+|g| T^{2}+|\nu| T} .
$$


The right-hand side is indeed integrable for all $(g, \nu) \in \mathbb{R}^{2}$, as long as $a>0$.

We define the infinite-volume two-point function and susceptibility by

$$
\left.G_{0, x}(a, g, \nu ; 0)=\lim _{N \rightarrow \infty} G_{N ; 0, x}(a, g, \nu ; 0), \quad \chi_{(} a, g, \nu ; 0\right)=\lim _{N \rightarrow \infty} \chi_{N}(a, g, \nu ; 0),
$$

assuming the limits exist.

\subsection{Supersymmetry and $n=0$}

The two-point function for the polymer model can be written exactly as the supersymmetric integral

$$
G_{N ; 0, x}(a, g, \nu ; 0)=\int e^{-\sum_{y \in \Lambda_{N}}\left(a \tau_{y}^{3}+g \tau_{y}^{2}+\nu \tau_{y}\right)} e^{-S} \bar{\phi}_{0} \phi_{x}
$$

with

$$
\tau_{y}=\phi_{y} \bar{\phi}_{y}+\psi_{y} \bar{\psi}_{y}, \quad S=\sum_{y \in \Lambda_{N}}\left(\phi_{y}(-\Delta \bar{\phi})_{y}+\psi_{y}(-\Delta \bar{\psi})_{y}\right)
$$

The above notation is explained in [8, Chapter 11] and the identity (1.14) is an immediate consequence of [8, Corollary 11.3.7]. The analysis of the supersymmetric model is a modification of the analysis of the $|\varphi|^{6}$ model, which follows the same well-trodden path as for the 4-dimensional analysis in $[4,35]$. Formulas for the spin system involving the number $n$ of spin components transfer to the polymer setting with $n=0$. For notational simplicity, we focus our discuss in this paper on the case $n \geq 1$, and comment occasionally on the supersymmetric case.

\subsection{Main result}

Our main result is Theorem 1.1. The existence of the limit defining the tricritical two-point function, namely the left-hand side of (1.17), is part of its statement. For $n \geq 1$ the point $\left(g_{c}(a), \nu_{c}(a)\right)$ is the tricritical point, and for $n=0$ it is the tricritical theta point. In terms of critical exponents, (1.17) says that tricritical two-point function has decay $|x|^{-(d-2+\eta)}$ with $\eta=0$.

Theorem 1.1. Let $d=3$ and $n \geq 0$. Let $L$ be sufficiently large and $\delta>0$ sufficiently small. There exists a continuous function $\left(g^{*}(t, a), \nu^{*}(t, a)\right)$ of $(t, a) \in(0, \delta)^{2}$, with limit $\left(g_{c}(a), \nu_{c}(a)\right)=$ $\lim _{t \downarrow 0}\left(g^{*}(t, a), \nu^{*}(t, a)\right)$, such that for $(t, a) \in(0, \delta)^{2}$ the limit

$$
G_{0, x}\left(a, g^{*}(t, a), \nu^{*}(t, a) ; n\right)=\lim _{N \rightarrow \infty} G_{N ; 0, x}\left(a, g^{*}(t, a), \nu^{*}(t, a) ; n\right)
$$

exists, and moreover

$$
\lim _{t \downarrow 0} G_{0, x}\left(a, g^{*}(t, a), \nu^{*}(t, a) ; n\right)=A_{a, n} \frac{1}{|x|}\left(1+O\left((\log |x|)^{-1}\right)\right)
$$

as $x \rightarrow \infty$, with $A_{a, n}=(4 \pi)^{-1}(1+O(a))$.

It would be of interest to study the geometry of the curve $\left(g^{*}(t, a), \nu^{*}(t, a)\right)$ as $t$ varies, but this has not yet been investigated. It would also be of interest to consider the approach to the tricritical point from a more general direction; see Section 1.5.1. 
Theorem 1.2. For $n \geq 0$, the asymptotic behaviour of the tricritical point $\left(\nu_{c}(a), g_{c}(a)\right)$, as a $\downarrow 0$, is given (with $\left.C_{0,0}=\left(-\Delta_{\mathbb{Z}^{3}}\right)_{0,0}^{-1}\right)$ by

$$
\begin{aligned}
& g_{c}(a)=-\frac{3}{2}(n+4) C_{0,0} a+O\left(a^{2}\right), \\
& \nu_{c}(a)=\frac{3}{4}(n+4)(n+2) C_{0,0}^{2}+O\left(a^{2}\right) .
\end{aligned}
$$

\subsection{Discussion}

\subsubsection{Conjectured phase diagram}

The conjectured phase diagram associated with the tricritical point, as predicted by the Landau mean-field theory (see, e.g., [1, Section 7.6.4] or [27, Appendix 5.A]), is illustrated in Figure 1.

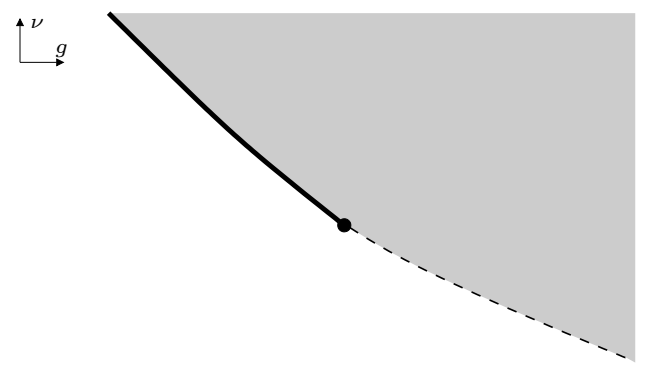

Figure 1: The conjectured phase diagram for $n \geq 0$. For $n \geq 1$, the tricritical point lies at the confluence of lines of first-order (solid) and second-order (dashed) phase transitions. For $n=0$, the dashed line corresponds to self-avoiding walk critical points, while the solid line corresponds to polymer collapse. The shaded region is the disordered phase.

A complete analysis of the phase diagram for the polymer model on the complete graph (meanfield theory) is given in [9]. We expect that the phase diagram on $\mathbb{Z}^{d}$ is qualitatively the same for all $d \geq 3$.

Theorem 1.1 indicates that Gaussian decay of the two-point function occurs at the point $\left(g_{c}(a), \nu_{c}(a)\right)$, but does not show that it is a tricritical point in the sense of being a point of confluence of first- and second-order transitions. However, for dimension $d=3$, we expect that the tricritical point is the only point in the $(g, \nu)$ plane where Gaussian decay occurs. A more complete description would require an analysis of the neighbourhood of $\left(g_{c}, \nu_{c}\right)$, a very difficult problem.

There is potential to extend our methods to study the divergence of the susceptibility (for $n \geq 0$ ) and specific heat (for $n \geq 1$ ) as the tricritical point is approached from the disordered phase. Our preliminary calculations support the conjecture that the open half plane

$$
H_{n}=\left\{(g, \nu) \in \mathbb{R}^{2}: \nu+(n+2) C_{0,0} g>0\right\}
$$

plays a key role for this. In particular, given a point $(g, \nu)$ such that $\left(g-g_{c}, \nu-\nu_{c}\right) \in H_{n}$, we conjecture that the susceptibility and specific heat are finite along the line segment $\left(g_{c}+t(g-\right.$ $\left.\left.g_{c}\right), \nu_{c}+t\left(\nu-\nu_{c}\right)\right)$ for $t \in\left(0, t_{*}\right]$ (for $t_{*}$ small depending on $a$ ), that the susceptibility asymptotically 
diverges as a multiple of $t^{-1}$ as $t \downarrow 0$, and that the specific heat asymptotically diverges as a multiple of $t^{-1 / 2}$. The conjecture assumes that $a$ is taken smaller as the angle of approach becomes closer to the boundary of $H_{n}$.

Our present methods are not sufficient to study the high-density phase. The related problem of the high-density phase of the weakly self-avoiding walk on a 4-dimensional hierarchical lattice has been studied in [21]. Recent progress on applying the RG to study broken symmetry was obtained in [29], where the critical magnetisation of the 4-dimensional $\varphi^{4}$ model is analysed.

\subsubsection{Infrared asymptotic freedom}

For dimension $d=4$, the mean-field $|x|^{-2}$ decay of the critical two-point function has been extensively studied $[4,19,20,35]$, both for spin and polymer (weakly self-avoiding walk) models. On $\mathbb{Z}^{4}$, the mean-field behaviour results from infrared asymptotic freedom, which is itself closely connected to the marginal nature (in the RG sense) of $|\varphi|^{4}$ for $d=4$. The RG flow is to a Gaussian fixed point. The logarithmic divergence of the massive bubble diagram $\sum_{x \in \mathbb{Z}^{4}}\left[\left(-\Delta+m^{2}\right)_{0, x}^{-1}\right]^{2}$ as $m^{2} \downarrow 0$ plays an important role, especially in the study of the divergence of thermodynamic quantities in the approach to the critical point where logarithmic corrections appear $[3,5,23]$.

For dimension $d=3,|\varphi|^{4}$ becomes a relevant monomial, whereas $|\varphi|^{6}$ is marginal. The $|x|^{-1}$ decay in (1.17) is again mean-field behaviour, which is again a consequence of infrared asymptotic freedom, and correspondingly a Gaussian RG fixed point. The role of the bubble diagram is now played instead by the logarithmically divergent diagram $\sum_{x \in \mathbb{Z}^{4}}\left[\left(-\Delta+m^{2}\right)_{0, x}^{-1}\right]^{3}$; the bubble diagram diverges as $m^{-1}$.

While both are governed by the Gaussian free field fixed point, a difference between the tricritical theory for $\mathbb{Z}^{3}$ and the critical theory for $\mathbb{Z}^{4}$ is that for the latter it is only required to tune one parameter (coefficient of $|\varphi|^{2}$ ) to obtain a critical theory, whereas for $\mathbb{Z}^{3}$ it is necessary to tune two parameters (coefficients of $|\varphi|^{2}$ and $|\varphi|^{4}$ ) to obtain the tricritical theory. This has long been understood in the physics literature, e.g., [36,38].

Much of our proof of Theorem 1.1 parallels the analysis used for the 4-dimensional case in $[4,35]$. In particular, with minor modifications, the results in $[15,16]$ provide the analysis of a single RG step. The tuning of parameters follows as in [7] with only notational changes. The analysis of perturbation theory is also similar to that in $[4,35]$, but here we present an improved and streamlined treatment of perturbation theory, which in particular demystifies the change of variables used in [6].

\subsubsection{Interacting self-avoiding walk}

The tricritical model of the theta point studied in this paper was investigated extensively in the physics literature during the 1980s, e.g., $[17,18]$ where misconceptions in the earlier literature were clarified. We are not aware of any previous mathematically rigorous analysis. In the mathematics literature, it has been more common to study the interacting self-avoiding walk, in which a selfavoiding or weakly self-avoiding walk receives an energetic reward for nearest-neighbour contacts [26]. The interacting self-avoiding walk has been studied in dimensions $d>4$ in $[22,37]$, in dimension $d=4$ in [10], and in dimension $d=1$ in [24,25]. The interacting prudent walk was studied in [33]. Recent numerical work for $d=2$ appears in [11]. 


\section{The RG map and RG flow}

Our RG method is based on a multiscale analysis which is implemented via the finite-range covariance decomposition discussed in Section 2.1. Representations for the susceptibility and the two-point function are presented in Section 2.2. In Section 2.3, the RG map is discussed along with the estimates on the global RG flow which are an essential ingredient for the proof of our main result. The tricritical point is identified in Section 2.4.

\subsection{Covariance decomposition and progressive integration}

We generalise (1.2) and define

$$
V_{a, g, \nu, z}\left(\varphi_{x}\right)=\frac{1}{8} a\left|\varphi_{x}\right|^{6}+\frac{1}{4} g\left|\varphi_{x}\right|^{4}+\frac{1}{2} \nu\left|\varphi_{x}\right|^{2}+\frac{1}{2} z \varphi_{x}(-\Delta \varphi)_{x}
$$

By definition, for any mass $m>0$ and for any wave function renormalisation $z_{0}>-1$,

$$
V_{a, g, \nu, 1}\left(\varphi_{x}\right)=V_{0,0, m^{2}, 1}\left(\left(1+z_{0}\right)^{-1 / 2} \varphi_{x}\right)+V_{a_{0}, g_{0}, \nu_{0}, z_{0}}\left(\left(1+z_{0}\right)^{-1 / 2} \varphi_{x}\right),
$$

with

$$
a_{0}=a\left(1+z_{0}\right)^{3}, \quad g_{0}=g\left(1+z_{0}\right)^{2}, \quad \nu_{0}=\left(1+z_{0}\right) \nu-m^{2} .
$$

For $X \subset \Lambda$, we define

$$
V_{0}(X)=V_{0}(\varphi, X)=\sum_{x \in X} V_{a_{0}, g_{0}, \nu_{0}, z_{0}}\left(\varphi_{x}\right), \quad Z_{0}(\varphi)=e^{-V_{0}\left(\varphi, \Lambda_{N}\right)} .
$$

Given a positive semi-definite covariance $\Lambda \times \Lambda$ matrix $C$, we write expectation with respect to the Gaussian measure for $n$-component fields as $\mathbb{E}_{C}$. For $n=0$, we instead use a superexpectation, exactly as in [5]. Let $C=\left(-\Delta_{\Lambda_{N}}+m^{2}\right)^{-1}$, where $\Lambda_{N}$ is the discrete torus of period $L^{N}$. For $m^{2}>0$, the inverse matrix exists and it is positive definite. Then, with $\tilde{F}(\varphi)=F\left(\left(1+z_{0}\right)^{1 / 2} \varphi\right)$, the change of variables $\varphi_{x} \mapsto\left(1+z_{0}\right)^{1 / 2} \varphi_{x}$ gives

$$
\langle F\rangle_{a, g, \nu, N}=\frac{\mathbb{E}_{C} \tilde{F} Z_{0}}{\mathbb{E}_{C} Z_{0}}
$$

We use decompositions of both of the covariances $\left(-\Delta_{\mathbb{Z}^{d}}+m^{2}\right)^{-1}$ and $\left(-\Delta_{\Lambda_{N}}+m^{2}\right)^{-1}$, based on the method of [2]. For $\mathbb{Z}^{d}$, this Green function exists for $d>2$ for all $m^{2} \geq 0$, but for finite $\Lambda$ we must restrict to $m^{2}>0$. As we discuss in Appendix A, there is a sequence $\left(C_{j}\right)_{1 \leq j<\infty}$ (depending on $m^{2} \geq 0$ ) of positive-definite covariances $C_{j}$ on $\mathbb{Z}^{d}$ such that

$$
\left(\Delta_{\mathbb{Z}^{d}}+m^{2}\right)^{-1}=\sum_{j=1}^{\infty} C_{j} \quad\left(m^{2} \geq 0\right) .
$$

The $C_{j}$ are translation invariant and have the finite-range property $C_{j ; x, y}=0$ if $|x-y| \geq \frac{1}{2} L^{j}$. Thus, for $j<N, C_{j}$ can also be identified as a covariance on the torus $\Lambda$. For $m^{2}>0$, there is also a covariance $C_{N, N}$ on $\Lambda$ such that

$$
\left(-\Delta_{\Lambda}+m^{2}\right)^{-1}=\sum_{j=1}^{N-1} C_{j}+C_{N, N} \quad\left(m^{2}>0\right),
$$


so the finite-volume and infinite-volume decompositions agree until the last term in the finitevolume decomposition. Properties of the covariance decomposition are collected in Appendix A.

We define the mass scale $j_{m}$ to be the largest integer $j$ such that $m L^{j} \leq 1$. In particular, $\lim _{m^{2} \downarrow 0} j_{m}=\infty$. By Proposition A.1, for multi-indices $\alpha$, $\beta$ with $\ell^{1}$ norms $|\alpha|_{1},|\beta|_{1}$ at most some fixed value $p$,

$$
\left|\nabla_{x}^{\alpha} \nabla_{y}^{\beta} C_{j ; x, y}\right| \leq c \vartheta_{j-1} L^{-(j-1)\left(2[\varphi]+\left(|\alpha|_{1}+|\beta|_{1}\right)\right)},
$$

where

$$
\vartheta_{j}=2^{-\left(j-j_{m}\right)_{+}}
$$

with $x_{+}=\max \{x, 0\}$, where the dimension of the field is

$$
[\varphi]=\frac{d-2}{2}=\frac{1}{2}
$$

and where the constant $c$ depends on $p$ but not on $m^{2}, j, L$. The bound (2.8) also holds for $C_{N, N}$ if $m^{2} L^{2(N-1)} \geq \delta$ for some fixed $\delta>0$, with $c$ depending on $\delta$ but not on $N$.

For $n \geq 1$, we write $\mathbb{E}_{C} \theta F$ for the convolution of $F$ with the Gaussian expectation $\mathbb{E}_{C}$. Explicitly, $\theta$ is the shift operator $\theta F(\varphi, \zeta)=F(\varphi+\zeta)$, and

$$
\left(\mathbb{E}_{C} \theta F\right)(\varphi)=\mathbb{E}_{C} F(\varphi+\zeta) .
$$

In (2.11), the expectation $\mathbb{E}_{C}$ acts on $\zeta$ with $\varphi$ held fixed. By a standard property of Gaussian integration, the decomposition (2.7) gives

$$
\mathbb{E}_{C} \theta F=\left(\mathbb{E}_{C_{N, N}} \theta \circ \mathbb{E}_{C_{N-1}} \theta \circ \cdots \circ \mathbb{E}_{C_{1}} \theta\right) F .
$$

With $Z_{0}=e^{-V_{0}\left(\Lambda_{N}\right)}$ as in $(2.4)$, we define

$$
Z_{j+1}=\mathbb{E}_{C_{j+1}} \theta Z_{j} \quad(j<N) .
$$

In particular,

$$
Z_{N}=\mathbb{E}_{C} \theta Z_{0}, \quad Z_{N}(0)=\mathbb{E}_{C} Z_{0}
$$

To simplify the notation, we write $\mathbb{E}_{j}=\mathbb{E}_{C_{j}}$, and leave implicit the dependence of $C_{j}$ on $m^{2}$. There is a supersymmetric version of (2.12)-(2.14), exactly as in [5].

\subsection{Susceptibility and two-point function}

\subsubsection{Susceptibility}

Let $n \geq 1$. We define

$$
\hat{\chi}_{N}\left(m^{2}, a_{0}, g_{0}, \nu_{0}, z_{0}\right)=\sum_{x \in \Lambda_{N}} \frac{\mathbb{E}_{C}\left(\zeta_{0}^{1} \zeta_{x}^{1} Z_{0}(\zeta)\right)}{\mathbb{E}_{C}\left(Z_{0}(\zeta)\right)} .
$$

By (2.5), when (2.3) is satisfied the finite volume susceptibility obeys

$$
\chi_{N}(a, g, \nu)=\left(1+z_{0}\right) \hat{\chi}_{N}\left(m^{2}, a_{0}, g_{0}, \nu_{0}, z_{0}\right) .
$$

Although (2.16) requires (2.3), it is nevertheless useful at times to relinquish the identity and consider the variables $\left(m^{2}, a_{0}, g_{0}, \nu_{0}, z_{0}\right)$ on the right-hand side of $(2.5)$ as independent variables. 
Given a test function $J: \Lambda_{N} \rightarrow \mathbb{R}^{n}$, let

$$
\Sigma_{N}(J)=\mathbb{E}_{C}\left(Z_{0}(\zeta) e^{(\zeta, J)}\right), \quad \text { where }(\zeta, J)=\sum_{x \in \Lambda_{N}} \sum_{i=1}^{n} \zeta_{x}^{i} J_{x}^{i}
$$

Differentiation in the direction $\mathbb{1}=(1,0, \ldots, 0)$ gives

$$
\hat{\chi}_{N}\left(m^{2}, a_{0}, g_{0}, \nu_{0}, z_{0}\right)=\frac{1}{\left|\Lambda_{N}\right|} \frac{D^{2} \Sigma_{N}(0 ; \mathbb{1}, \mathbb{1})}{Z_{N}(0)} .
$$

By completing the square, we obtain

$$
\Sigma_{N}(J)=e^{\frac{1}{2}(J, C J)} \mathbb{E}_{C}\left(Z_{0}(\zeta+C J)\right)=e^{\frac{1}{2}(J, C J)} Z_{N}(C J) .
$$

Differentiation of (2.19), together with $C \mathbb{1}=m^{-2} \mathbb{1}$, leads to

$$
D^{2} \Sigma_{N}(0 ; \mathbb{1}, \mathbb{1})=\frac{1}{m^{2}}\left|\Lambda_{N}\right| Z_{N}(0)+\frac{1}{m^{4}} D^{2} Z_{N}(0 ; \mathbb{1}, \mathbb{1}),
$$

and hence

$$
\hat{\chi}_{N}\left(m^{2}, a_{0}, g_{0}, \nu_{0}, z_{0}\right)=\frac{1}{m^{2}}+\frac{1}{m^{4}} \frac{1}{\left|\Lambda_{N}\right|} \frac{D^{2} Z_{N}(0 ; \mathbb{1}, \mathbb{1})}{Z_{N}(0)} .
$$

Thus the evaluation of the susceptibility reduces to the evaluation of $Z_{N}(\varphi)$ for a constant field $\varphi$.

\subsubsection{Two-point function and observable fields}

Let $n \geq 1$. By (2.5), when (2.3) is satisfied the two-point function (1.5) can be written as

$$
G_{N ; \times, y}(a, g, \nu ; n)=\left(1+z_{0}\right) \frac{\mathbb{E}_{C}\left(\zeta_{\mathrm{x}}^{1} \zeta_{\mathrm{y}}^{1} Z_{0}(\zeta)\right)}{\mathbb{E}_{C} Z_{0}(\zeta)}
$$

Let $\hat{1}=n^{-1 / 2}(1, \ldots, 1) \in \mathbb{R}^{n}$. Given $\mathrm{x}, \mathrm{y} \in \mathbb{Z}^{d}$ and given observable fields $\sigma_{\mathrm{x}}, \sigma_{\mathrm{y}} \in \mathbb{R}$, we define $V_{0}$ by

$$
V_{0 ; x}=V_{0 ; x}^{\varnothing}-\sigma_{\mathrm{x}}\left(\hat{1} \cdot \varphi_{\mathrm{x}}\right) \mathbb{1}_{x=\mathrm{x}}-\sigma_{\mathrm{y}}\left(\hat{1} \cdot \varphi_{\mathrm{y}}\right) \mathbb{1}_{x=\mathrm{y}},
$$

with $V_{0}^{\varnothing}$ a new notation for the bulk polynomial (2.4). Although $\sigma_{\mathrm{x}}$ and $\sigma_{\mathrm{y}}$ carry subscripts $\mathrm{x}, \mathrm{y}$, they are real constants. Let $\partial_{\sigma_{x} \sigma_{y}}^{2}$ denote $\left.\frac{\partial^{2}}{\partial \sigma_{x} \sigma_{y}}\right|_{\sigma_{x}=\sigma_{y}=0}$. Then

$$
G_{N ; \times, y}(a, g, \nu ; n)=\left(1+z_{0}\right) \partial_{\sigma_{\mathrm{y}} \sigma_{\mathrm{y}}}^{2} \log \mathbb{E}_{C} e^{-V_{0}(\Lambda)}
$$

The observable field $\sigma_{x} \hat{1} \mathbb{1}_{x=x}+\sigma_{y} \hat{1} \mathbb{1}_{x=y}$ can be regarded as an implementation of a special case of the test function $J$ used in (2.17). However, for the susceptibility only a constant test function was needed, whereas the observable fields are highly localised. For the two-point function, we now regard the observable field as part of the potential $V$, and we track the flow of the new terms in $V$ under progressive integration. It is via this flow that we will be able to compute the behaviour of the two-point function. 
A hybrid approach is also possible, as follows. Let $\partial_{\sigma_{\mathrm{x}}}$ denote $\left.\frac{\partial}{\partial \sigma_{\mathrm{x}}}\right|_{\sigma_{\mathrm{x}}=\sigma_{\mathrm{y}}=0}$ and $Z_{N}^{\varnothing}(0)=$ $\mathbb{E}_{C} e^{-V_{0}^{\varnothing}(\Lambda)}$. Let $\hat{\mathbb{1}}$ denote the constant field $\hat{\mathbb{1}}_{x}=\hat{1}$ for all $x \in \Lambda$. We note for later use that, with $Z_{0}$ now defined using $(2.23)$,

$$
\hat{\chi}_{N}=\frac{\partial_{\sigma_{\mathrm{x}}} D \Sigma_{N}(0 ; \hat{\mathbb{1}})}{Z_{N}^{\varnothing}(0)}=\frac{\partial_{\sigma_{\mathrm{x}}} D Z_{N}(0 ; C \hat{\mathbb{1}})}{Z_{N}^{\varnothing}(0)}=\frac{1}{m^{2}} \frac{\partial_{\sigma_{\mathrm{x}}} D Z_{N}(0 ; \hat{\mathbb{1}})}{Z_{N}^{\varnothing}(0)},
$$

where the second equality follows from (2.19) and $C \mathbb{1}=m^{-2} \mathbb{1}$.

The calculation of the two-point function only requires the second derivative in (2.24), and therefore only needs the dependence on the observables to second order. As in $[4,16,35]$, we formalise this simplification via use of a quotient space, in which two functions of $\varphi, \sigma_{\mathrm{x}}, \sigma_{\mathrm{y}}$ become equivalent if their formal power series in the observable fields agree to order $1, \sigma_{\mathrm{x}}, \sigma_{\mathrm{y}}, \sigma_{\mathrm{x}} \sigma_{\mathrm{y}}$. Thus we define $\mathcal{N}$ to consist of $C^{16}$ functions of $\varphi$ of the form

$$
F=F^{\varnothing}+\sigma_{\mathrm{x}} F^{\mathrm{x}}+\sigma_{\mathrm{y}} F^{\mathrm{y}}+\sigma_{\mathrm{x}} \sigma_{\mathrm{y}} F^{\mathrm{xy}},
$$

where each $F^{\alpha}$ is a function of $\varphi$.

For $n=0$, the above can be modified exactly as in [4], with observable fields $\sigma_{\mathrm{x}}, \sigma_{\mathrm{y}} \in \mathbb{C}$, and

$$
V_{0 ; x}=V_{0 ; x}^{\varnothing}-\sigma_{\mathrm{x}} \bar{\phi}_{\mathrm{x}} \mathbb{1}_{x=\mathrm{x}}-\bar{\sigma}_{\mathrm{y}} \phi_{\mathrm{y}} \mathbb{1}_{x=\mathrm{y}} .
$$

Similarly to (2.24), since the partition function with $\sigma_{\mathrm{x}}=\bar{\sigma}_{\mathrm{y}}=0$ is equal to 1 by supersymmetry, with $\mathbb{E}_{C}$ now a super-expectation we have

$$
G_{N ; \times, y}(a, g, \nu ; 0)=\left(1+z_{0}\right) \partial_{\sigma_{\times} \bar{\sigma}_{y}}^{2} \mathbb{E}_{C} e^{-V_{0}(\Lambda)}=\left(1+z_{0}\right) \partial_{\sigma_{\times} \bar{\sigma}_{\mathrm{y}}}^{2} \log \mathbb{E}_{C} e^{-V_{0}(\Lambda)}
$$

\subsection{RG map}

Integration of a single scale is recorded in (2.13) as $Z_{j+1}=\mathbb{E}_{C_{j+1}} \theta Z_{j}$, with $Z_{0}=e^{-V_{0}(\Lambda)}$. Now we use the version (2.23) of $V_{0}$ with observables. It would be desirable to have $Z_{j}$ also represented by an effective potential $V_{j}$ as $Z_{j} \approx e^{-V_{j}(\Lambda)}$ with $V_{j}$ of the same form as $V_{0}$ but with renormalised coupling constants. However, such an approximation requires great care. Instead, exactly as in [16], we use a representation involving the circle product. In the following, we do not always give precise definitions, as these can be found in [16, Section 1] with the same notation as used here.

We need the following definitions. For each $j=0,1, \ldots, N$, the discrete torus $\Lambda_{N}$ of period $L^{N}$ partitions into $L^{N-j}$ disjoint $d$-dimensional cubes of side $L^{j}$, called blocks, or $j$-blocks. We denote the set of $j$-blocks by $\mathcal{B}_{j}$. A union of $j$-blocks (possibly empty) is called a polymer or $j$-polymer, and the set of $j$-polymers is denoted $\mathcal{P}_{j}$. The set of blocks in a polymer $X \in \mathcal{P}_{j}$ is denoted $\mathcal{B}_{j}(X)$, and the set of connected components $\mathcal{C}_{j}(X)$. The unique $N$-block is $\Lambda_{N}$ itself. With these definitions, we write each $Z_{j}$ in the form

$$
Z_{j}=e^{\xi_{j}}\left(I_{j} \circ K_{j}\right)(\Lambda)=e^{\xi_{j}} \sum_{X \in \mathcal{P}_{j}} I_{j}(\Lambda \backslash X) K_{j}(X),
$$

where $I_{j}(X)$ and $K_{j}(X)$ are functions of the field in the neighbourhood of $X$, and the prefactor has the form

$$
\xi_{j}=-u_{j}|\Lambda|+\frac{1}{2}\left(q_{\mathrm{x}, j}+q_{\mathrm{y}, j}\right) \sigma_{\mathrm{x}} \sigma_{\mathrm{y}}
$$


The functions $I_{j}$ and $K_{j}$ satisfy the factorisation properties $I_{j}(X)=\prod_{B \in \mathcal{B}_{j}(X)} I_{j}(B)$ and $K_{j}(X)=$ $\prod_{C \in \mathcal{C}_{j}(X)} K_{j}(C)$. More precisely, $I_{j}(B)=e^{-V_{j}(B)}\left(1+W_{j}\left(V_{j}, B\right)\right)$, where $W_{j}\left(V_{j}, B\right)$ is an explicit quadratic function (defined in (3.16) below) of

$$
V_{j, x}=a_{j} \tau_{x}^{3}+g_{j} \tau_{x}^{2}+\nu_{j} \tau_{x}+z_{j} \tau_{\Delta, x}-\lambda_{\mathrm{x}, j} \sigma_{\mathrm{x}}\left(\hat{1} \cdot \varphi_{x}\right) \mathbb{1}_{x=\mathrm{x}}-\lambda_{\mathrm{y}, j} \sigma_{\mathrm{y}}\left(\hat{1} \cdot \varphi_{x}\right) \mathbb{1}_{x=\mathrm{y}} .
$$

The nonperturbative coordinate $K_{j}$ encompasses all irrelevant (in RG sense) terms that are $O\left(V_{j}^{3}\right)$. All first- and second-order contributions are in $I_{j}$. Since $W_{j}\left(V_{j}\right)=O\left(V_{j}^{2}\right)$, it is instructive to pretend that $I_{j}(X) \approx e^{-V_{j}(X)}$. At the last scale, (2.29) becomes a sum over the two polymers $X=\varnothing$ and $X=\Lambda_{N}$, and hence

$$
Z_{N}=e^{\xi_{N}}\left(I_{N}\left(\Lambda_{N}\right)+K_{N}\left(\Lambda_{N}\right)\right)=e^{\xi_{N}}\left[e^{-V_{N}\left(\Lambda_{N}\right)}\left(1+W_{N}\left(\Lambda_{N}\right)\right)+K_{N}\left(\Lambda_{N}\right)\right] .
$$

If the above can be achieved with $K_{N}$ appropriately vanishing in the limit $N \rightarrow \infty$, then we would have $Z_{N}(\varphi) \approx e^{\xi_{N}-V_{N}(\varphi)}$, and differentiation as in (2.21) and (2.24) would permit the susceptibility and two-point function to be evaluated as

$$
\chi_{N} \approx\left(1+z_{0}\right)\left(m^{-2}+m^{-4} \nu_{N}\right), \quad G_{N ; \times, y} \approx\left(1+z_{0}\right) q_{N} .
$$

This suggests that knowledge of the coupling constants $\nu_{N}$ and $q_{N}$ is tantamount to a computation of the susceptibility and two-point function.

The calculation of the coupling constants requires an understanding of iterations of the $R G$ map, which is a map

$$
\left(V_{j}, K_{j}\right) \mapsto\left(U_{j+1}, K_{j+1}\right)=\left(\delta \xi_{j+1}, V_{j+1}, K_{j+1}\right)
$$

that is defined in such a way that

$$
Z_{j+1}=\mathbb{E}_{j+1} \theta Z_{j}=e^{\xi_{j}} \mathbb{E}_{j+1} \theta\left(I_{j} \circ K_{j}\right)(\Lambda)=e^{\xi_{j+1}}\left(I_{j+1} \circ K_{j+1}\right)(\Lambda),
$$

with

$$
\delta \xi_{j+1}=\xi_{j+1}-\xi_{j}=-\delta u_{j+1}|\Lambda|+\frac{1}{2}\left(\delta q_{x, j}+\delta q_{\mathrm{y}, j}\right) \sigma_{\mathrm{x}} \sigma_{\mathrm{y}} .
$$

Indefinite iteration of the $\mathrm{RG}$ map requires tuning of the initial values $g_{0}, \nu_{0}$ to values corresponding to the tricritical point.

The map $\left(V_{j}, K_{j}\right) \mapsto\left(\delta \xi_{j+1}, V_{j+1}\right)$ is a combination of a perturbative map $\mathrm{PT}$, which is a function only of $V_{j}$, and a nonperturbative remainder which extracts the relevant part of $K_{j}$. Thus,

$$
\begin{aligned}
V_{j+1} & =\mathrm{PT}_{j}^{(0)}\left(V_{j}\right)+R_{j+1}^{(0)}\left(V_{j}, K_{j}\right), \\
\delta \xi_{j+1} & =\mathrm{PT}_{j}^{(\xi)}\left(V_{j}\right)+R_{j+1}^{(\xi)}\left(V_{j}, K_{j}\right) .
\end{aligned}
$$

The maps $\mathrm{PT}_{j}^{(0)}, \mathrm{PT}_{j}^{(\xi)}$ are components of an explicit quadratic map $\mathrm{PT}_{j}$ defined in Section 3.2. [In detail, $\mathrm{PT}^{(0)}$ is the operator PT followed by the replacement of $\left(y, z, u, q_{\mathrm{x}}, q_{\mathrm{y}}\right)$ by $(0, y+z, 0,0,0)$, and $\mathrm{PT}^{(\xi)}$ consists of the $\left(u, q_{\mathrm{x}}, q_{\mathrm{y}}\right)$ components of PT.] We write the individual coefficients of the remainder terms as $R_{j+1}^{a}, R_{j+1}^{g}, \ldots$..

The next theorem is for the bulk, which corresponds to setting $\sigma_{\mathrm{x}}=\sigma_{\mathrm{y}}=0$. It asserts the existence of a global bulk RG flow with estimates on $K_{j}$ and on the remainders $R_{j}$ to perturbation 
theory. The statement of the theorem involves seminorms $\|F\|_{T_{0}\left(\ell_{j}\right)}$ (defined in $[3$, Section 2.3] for $n \geq 1$ and in [5, Section 6.3] for $n=0$ ). Although the proof of the theorem requires working with a stronger norm (see Section B.1.1), for our application we only need to know that

$$
|F(0)|+L^{-[\varphi] j}|D F(0 ; \mathbb{1})|+L^{-2[\varphi] j}\left|D^{2} F(0 ; \mathbb{1}, \mathbb{1})\right| \leq O(1)\|F\|_{T_{0}\left(\ell_{j}\right)},
$$

where $D$ denotes the derivative with respect to $\varphi$ in the direction $\mathbb{1}$ as in (2.18), as well as that if $R^{\varnothing}=R^{a}|\varphi|^{6}+R^{g}|\varphi|^{4}+R^{\nu}|\varphi|^{2}+R^{z}(\varphi \cdot(-\Delta \varphi))+R^{u}$ then

$$
\left|R_{j}^{a}\right|,\left|R_{j}^{z}\right|, L^{j}\left|R_{j}^{g}\right|, L^{2 j}\left|R_{j}^{\nu}\right|, L^{3 j}\left|R_{j}^{u}\right| \leq O(1)\left\|R^{\varnothing}(B)\right\|_{T_{0}\left(\ell_{j}\right)} .
$$

Theorem 2.1. Fix $L$ sufficiently large and $\delta>0$ sufficiently small. There are continuous functions $g_{0}^{c}, \nu_{0}^{c}, z_{0}^{c}$ of $\left(m^{2}, a_{0}\right) \in[0, \delta)^{2}$ such that if $\left(g_{0}, \nu_{0}, z_{0}\right)=\left(g_{0}^{c}\left(m^{2}, a_{0}\right), \nu_{0}^{c}\left(m^{2}, a_{0}\right), z_{0}^{c}\left(m^{2}, a_{0}\right)\right)$ then, for all $j<N$,

$$
L^{j}\left|g_{j}\right|, L^{2 j}\left|\nu_{j}\right|, L^{3 j}\left|\delta u_{j}\right|=O\left(\vartheta_{j} a_{j}\right),
$$

and the following remainder bounds hold, for $B \in \mathcal{B}_{j}, X \in \mathcal{C}_{j}$, and some (small) $\alpha>0$,

$$
\begin{aligned}
\left\|R_{j}^{\varnothing}(B)\right\|_{T_{0}\left(\ell_{j}\right)} & =O\left(\vartheta_{j}^{3} a_{j}^{3}\right) \\
\left\|W_{j}^{\varnothing}(B)\right\|_{T_{0}\left(\ell_{j}\right)} & =O\left(\vartheta_{j}^{2} a_{j}^{2}\right) \\
\left\|K_{j}^{\varnothing}(X)\right\|_{T_{0}\left(\ell_{j}\right)} & =O\left(\vartheta_{j} a_{j}\right)^{3+\alpha\left(\left|\mathcal{B}_{j}(X)\right|-2^{d}\right)_{+} .}
\end{aligned}
$$

The functions $g_{0}^{c}, \nu_{0}^{c}, z_{0}^{c}$ are $O\left(a_{0}\right)$, are equal to zero at $\left(m^{2}, 0\right)$, and obey $\frac{\partial g_{0}^{c}}{\partial a_{0}}, \frac{\partial \nu_{0}^{c}}{\partial a_{0}}, \frac{\partial z_{0}^{c}}{\partial a_{0}}=O(1)$ uniformly in $\left(m^{2}, a_{0}\right) \in[0, \delta)^{2}$. Moreover, all the above bounds hold also for $j=N$ provided $m^{2} \geq$ $L^{-2 N}$. Finally, the coupling constants $a_{j}, g_{j}, \nu_{j}, z_{j}$ and their remainder terms $R_{j}^{\varnothing}$ are independent of the volume parameter $N$ provided $N \geq j$.

Upper bounds in Theorem 2.1 are expressed in terms of $a_{j}$, so it is important to understand the behaviour of this sequence. As we discuss in detail in Section 4.1 below, $a_{j}$ obeys the recursion

$$
a_{j+1}=a_{j}-\beta_{j} a_{j}^{2}+O\left(\vartheta_{j}^{3} a_{j}^{3}\right)
$$

with explicit coefficients $\beta_{j}$. When $m^{2}>0, a_{j}$ converges to a constant which vanishes logarithmically as $m^{2} \downarrow 0$, and $a_{j} \rightarrow 0$ when $m^{2}=0$. This vanishing of the massless limit goes by the name of infrared asymptotic freedom and is a manifestation of the fact that the RG flows to a Gaussian fixed point.

The next theorem supplements Theorem 2.1 to permit nonzero values of the observable fields. It requires the following definition. Given $\mathrm{x}, \mathrm{y} \in \Lambda$, we define the coalescence scale $j_{\mathrm{xy}}$ to be the unique integer such that

$$
\frac{1}{2} L^{j_{x y}} \leq|\mathrm{x}-\mathrm{y}|<\frac{1}{2} L^{j_{x y}+1}
$$

namely $j_{\mathrm{xy}}=\left\lfloor\log _{L}(2|\mathrm{x}-\mathrm{y}|)\right\rfloor$. It follows from the finite-range property of $C_{j}$ that $C_{j ; \times, \mathrm{y}}=0$ if $j \leq j_{\mathrm{xy}}$.

Theorem 2.2. Fix $L$ sufficiently large and $\delta>0$ sufficiently small, and let $\left(m^{2}, a_{0}\right) \in[0, \delta)^{2}$. Let $\left(g_{0}, \nu_{0}, z_{0}\right)=\left(g_{0}^{c}\left(m^{2}, a_{0}\right), \nu_{0}^{c}\left(m^{2}, a_{0}\right), z_{0}^{c}\left(m^{2}, a_{0}\right)\right)$. Then, for all $j<N$ and for $x=\mathrm{x}, \mathrm{y}$, the 
following remainder bounds hold, for $B \in \mathcal{B}_{j}, X \in \mathcal{C}_{j}$, and some (small) $\alpha>0$ :

$$
\begin{aligned}
& \left|R_{j}^{\lambda_{x}}\right|=\mathbb{1}_{j<j_{x y}} O\left(\vartheta_{j} a_{j}^{2}\right), \\
& \left|R_{j}^{q_{x}}\right|=\mathbb{1}_{j \geq j_{x y}}|\mathrm{x}-\mathrm{y}|^{-2[\varphi]} 4^{-\left(j-j_{x y}\right)} O\left(\vartheta_{j} a_{j}\right), \\
& \left\|W_{j}^{x}(B)\right\|_{T_{0}\left(\ell_{j}\right)}=L^{-\left(j \wedge j_{x y}\right)[\varphi]} 2^{-\left(j-j_{x y}\right)}+O\left(\vartheta_{j}^{2} a_{j}^{2}\right), \\
& W_{j}^{\mathrm{xy}}(B)=0, \\
& \left\|K_{j}^{x}(X)\right\|_{T_{0}\left(\ell_{j}\right)}=L^{-\left(j \wedge j_{x y}\right)[\varphi]} 2^{-\left(j-j_{x y}\right)+} O\left(\vartheta_{j} a_{j}\right)^{2+\alpha\left(\left|\mathcal{B}_{j}(X)\right|-2^{d}\right)_{+}}, \\
& \left\|K_{j}^{\mathrm{xy}}(X)\right\|_{T_{0}\left(\ell_{j}\right)}=L^{-2\left(j \wedge j_{x y}\right)[\varphi]} 4^{-\left(j-j_{x y}\right)+} O\left(\vartheta_{j} a_{j}\right)^{1+\alpha\left(\left|\mathcal{B}_{j}(X)\right|-2^{d}\right)_{+}} .
\end{aligned}
$$

Moreover, all the above bounds hold also for $j=N$ provided $m^{2} \geq L^{-2 N}$. Finally, the coupling constants $\lambda_{x, j}, q_{x, j}$ and their remainder terms $R_{j}^{\lambda_{x}}, R_{j}^{q_{x}}$ are independent of the volume parameter $N$ provided $N \geq j$.

The bounds (2.42)-(2.44) and (2.47)-(2.52) imply that the leading order contributions to the two-point function and susceptibility are given by perturbation theory, i.e., by the map PT. Our main focus is therefore on the analysis of the map PT. The proof of Theorems 2.1-2.2 is discussed in Appendix B. It relies significantly on external results adapted from the 4-dimensional setting.

\subsection{Identification of tricritical point}

In this section, we identify the tricritical point.

Given $\left(m^{2}, a_{0}\right)$, Theorem 2.1 provides an initial condition for a global flow with final conditions $g_{\infty}=0$ and $\nu_{\infty}=0$ and with good bounds on $\left(V_{j}, K_{j}\right)$. This allows for an exact computation of $\hat{\chi}$ in the following corollary to Theorem 2.1.

Corollary 2.3. For $\left(m^{2}, a_{0}\right) \in(0, \delta)^{2}$,

$$
\hat{\chi}\left(m^{2}, a_{0}, g_{0}^{c}\left(a_{0}, m^{2}\right), \nu_{0}^{c}\left(a_{0}, m^{2}\right), z_{0}^{c}\left(a_{0}, m^{2}\right)\right)=\frac{1}{m^{2}} .
$$

Proof. In this proof, we take $\sigma_{\mathrm{x}}=\sigma_{\mathrm{y}}=0$. According to (2.21),

$$
\hat{\chi}_{N}\left(m^{2}, a_{0}, g_{0}, \nu_{0}, z_{0}\right)=\frac{1}{m^{2}}+\frac{1}{m^{4}} \frac{1}{\left|\Lambda_{N}\right|} \frac{D^{2} Z_{N}(0 ; \mathbb{1}, \mathbb{1})}{Z_{N}(0)},
$$

with $Z_{N}$ given by the sum in (2.32). It follows from Theorem 2.1 that the contributions from $W_{N}$ and $K_{N}$ vanish in the limit $N \rightarrow \infty$, and direct computation gives $D^{2} e^{-V_{N}}(0 ; \mathbb{1} \mathbb{1})=-\nu_{N}\left|\Lambda_{N}\right|$. Then (2.53) follows from the fact that $\lim _{N \rightarrow \infty} \nu_{N}=0$ by (2.41).

On the eight variables $a, g, \nu, m^{2}, a_{0}, g_{0}, \nu_{0}, z_{0}$, we impose the three constraints

$$
a_{0}=a\left(1+z_{0}\right)^{3}, \quad g_{0}=g\left(1+z_{0}\right)^{2}, \quad \nu_{0}=\left(1+z_{0}\right) \nu-m^{2},
$$

of (2.3), and the three constraints

$$
g_{0}=g_{0}^{c}\left(m^{2}, a_{0}\right), \quad \nu_{0}=\nu_{0}^{c}\left(m^{2}, a_{0}\right), \quad z_{0}=z_{0}^{c}\left(m^{2}, a_{0}\right),
$$

with $g_{0}^{c}, \nu_{0}^{c}, z_{0}^{c}$ the functions of Theorem 2.1. The next proposition shows that if we fix $\left(m^{2}, a\right)$ then the other six variables are determined by the constraints. 
Proposition 2.4. There exists $\delta_{1}>0$ and continuous functions $\left(g^{*}, \nu^{*}, a_{0}^{*}, g_{0}^{*}, \nu_{0}^{*}, z_{0}^{*}\right)$ of $\left(m^{2}, a\right) \in$ $\left[0, \delta_{1}\right)^{2}$, such that $(2.55)-(2.56)$ hold and

$$
a_{0}^{*}=a+O\left(a^{2}\right), \quad g_{0}^{*}, \nu_{0}^{*}, z_{0}^{*}=O(a) .
$$

Proof. For $\left(m_{0}^{2}, a_{0}\right) \in[0, \delta)^{2}$, set

$$
t\left(m^{2}, a_{0}\right)=\frac{a_{0}}{\left(1+z_{0}^{c}\left(m^{2}, a_{0}\right)\right)^{3}}
$$

By Theorem 2.1, $g_{0}^{c}, \nu_{0}^{c}, z_{0}^{c}$ are continuous in $\left(m^{2}, a_{0}\right) \in[0, \delta)^{2}$, are $O\left(a_{0}\right)$, and have bounded $a_{0^{-}}$ derivatives. Thus, with derivatives evaluated at $\left(m^{2}, a_{0}\right)$,

$$
\frac{\partial t}{\partial a_{0}}=\frac{\left(1+z_{0}^{c}\right)^{3}-3 a_{0}\left(1+z_{0}^{c}\right)^{2} \frac{\partial z_{0}^{c}}{\partial a_{0}}}{\left(1+z_{0}^{c}\right)^{6}}=1+O\left(a_{0}\right)>0 .
$$

For sufficiently small $\delta>0, t$ is therefore a strictly increasing continuous function of $a_{0} \in[0, \delta)$ such that $\left|t\left(m^{2}, u\right)-t\left(m^{2}, v\right)\right| \geq(1-O(\delta))|u-v|$ and hence, for $m^{2}, s$ fixed, $t\left(m^{2}, \cdot\right)$ is a continuously invertible map from $[0, \delta)$ onto the interval $\left[0, t\left(m^{2}, \delta\right)\right)$. We denote the inverse map as $a_{0}^{*}$, set

$$
g_{0}^{*}\left(m^{2}, a\right)=g_{0}^{c}\left(m^{2}, a_{0}^{*}\left(m^{2}, a\right)\right), \quad \nu_{0}^{*}\left(m^{2}, a\right)=\nu_{0}^{c}\left(m^{2}, a_{0}^{*}\left(m^{2}, a\right)\right), \quad z_{0}^{*}\left(m^{2}, a\right)=z_{0}^{c}\left(m^{2}, a_{0}^{*}\left(m^{2}, a\right)\right),
$$

and define

$$
g^{*}\left(m^{2}, a\right)=\frac{g_{0}^{*}}{\left(1+z_{0}^{*}\right)^{2}}, \quad \nu^{*}\left(m^{2}, a\right)=\frac{\nu_{0}^{*}+m^{2}}{1+z_{0}^{*}} .
$$

Since $\nu_{0}^{c}, z_{0}^{c}, a^{*}$ are continuous, it is also the case that $g_{0}^{*}, \nu_{0}^{*}, z_{0}^{*}, \nu^{*}$ are continuous. It is immediate that (2.55)-(2.56) hold, and also that (2.57) holds.

Let $\delta_{1}=\frac{1}{2} \delta$. By $(2.58),\left[0, \delta_{1}\right)$ lies in the intersection over $m^{2}>0$ of the intervals $\left[0, t\left(m^{2}, \delta\right)\right)$. Therefore, $a=t\left(m^{2}, a_{0}\right)$ can be solved for $a_{0}$ as a function $a_{0}^{*}\left(m^{2}, a\right)$ for $a \in\left[0, \delta_{1}\right)$ and $a_{0}^{*}$ is continuous in $a$ for $m^{2}$ fixed. To see that $a_{0}^{*}$ is jointly continuous in $\left(m^{2}, a\right)$, it suffices to show that if $\left(\hat{m}^{2}, \hat{a}\right) \rightarrow\left(m^{2}, a\right)$ then $\hat{a}_{0} \rightarrow a_{0}$, where $\hat{a}_{0}, a_{0}$ solve $t\left(\hat{m}^{2}, \hat{a}_{0}\right)-\hat{a}=0=t\left(m^{2}, a_{0}\right)-a$. This follows from $\left(1-O\left(a_{*}\right)\right)\left|\hat{a}_{0}-a_{0}\right| \leq\left|t\left(\hat{m}^{2}, \hat{a}_{0}\right)-t\left(\hat{m}^{2}, a_{0}\right)\right|=\left|\left(t\left(m^{2}, a_{0}\right)-t\left(\hat{m}^{2}, a_{0}\right)\right)+(\hat{a}-a)\right| \rightarrow 0$, since $t\left(\cdot, a_{0}\right)$ is continuous by $(2.58)$ and the continuity of $z_{0}^{c}$.

With the continuous functions produced in Proposition 2.4, we see from (2.53) that

$$
\chi\left(a, g^{*}, \nu^{*}\right)=\left(1+z_{0}^{*}\right) \hat{\chi}\left(m^{2}, a_{0}^{*}, g_{0}^{*}, \nu_{0}^{*}, z_{0}^{*}\right)=\left(1+z_{0}^{*}\right) \frac{1}{m^{2}} .
$$

In particular, the susceptibility $\chi\left(a, g^{*}, \nu^{*}\right)$ is finite if $m^{2}>0$, whereas $\chi\left(a, g^{*}, \nu^{*}\right) \rightarrow \infty$ as $m^{2} \downarrow 0$. The divergence of the susceptibility, together with the fact that the RG fixed point is Gaussian, leads us to define the tricritical point as

$$
\left(g_{c}(a), \nu_{c}(a)\right)=\left(g^{*}(0, a), \nu^{*}(0, a)\right) .
$$

We will see in Section 5.1 that $\left(g^{*}\left(m^{2}, a\right), \nu^{*}\left(m^{2}, a\right)\right)$ defines the curve of Theorem 1.1, parametrised by $\mathrm{m}^{2}$. The geometry of the curve is of interest, but we do not investigate it in this paper. 


\section{The map PT and the approximate flow}

In this section, we define the perturbative map PT and its simplification called the approximate flow. In particular, we incorporate improvements to the treatment of the map PT used in $[3,6]$, and extend it to include a $|\varphi|^{6}$ term. The improvements include a more systematic treatment of the change of variables (transformation) used in [6], as well as the use of general estimates for coefficients arising in flow equations rather than detailed individual estimates based on explicit formulas. The main result of this section is Proposition 3.5, which provides the approximate flow.

We use the notation appropriate for $n \geq 1$. The relevant $\left(1, \tau, \tau^{2}\right)$ and marginal $\left(\tau^{3}, \tau_{\Delta}, \tau_{\nabla \nabla}\right)$ bulk monomials obeying Euclidean and $O(n)$ symmetry are:

$$
\begin{aligned}
1, \quad \tau=\frac{1}{2}|\varphi|^{2}, \quad \tau^{2} & =\frac{1}{4}|\varphi|^{4}, \quad \tau^{3}=\frac{1}{8}|\varphi|^{6}, \\
\tau_{\Delta}=\frac{1}{2} \varphi \cdot(-\Delta \varphi), \quad \tau_{\nabla \nabla} & =\frac{1}{4} \sum_{e \in \mathbb{Z}^{d}:|e|_{1}=1} \nabla^{e} \varphi \cdot \nabla^{e} \varphi .
\end{aligned}
$$

The following complex vector spaces of polynomials play a role:

$$
\begin{aligned}
\mathcal{V}^{\varnothing} & =\left\{a \tau^{3}+g \tau^{2}+\nu \tau+z \tau_{\Delta}: a, g, \nu, z \in \mathbb{C}\right\}, \\
\mathcal{U}^{\varnothing} & =\left\{V+y \tau_{\nabla \nabla}+u: V \in \mathcal{V}^{\varnothing}, y, u \in \mathbb{C}\right\}, \\
\mathcal{V} & =\left\{V-\lambda_{\mathrm{x}} \sigma_{\mathrm{x}}(\hat{1} \cdot \varphi) \mathbb{1}_{\mathrm{x}}-\lambda_{\mathrm{y}} \sigma_{\mathrm{y}}(\hat{1} \cdot \varphi) \mathbb{1}_{\mathrm{y}}: V \in \mathcal{V}^{\varnothing}, \lambda_{\mathrm{x}}, \lambda_{\mathrm{y}} \in \mathbb{C}\right\}, \\
\mathcal{U} & =\left\{U-\lambda_{\mathrm{x}} \sigma_{\mathrm{x}}(\hat{1} \cdot \varphi) \mathbb{1}_{\mathrm{x}}-\lambda_{\mathrm{y}} \sigma_{\mathrm{y}}(\hat{1} \cdot \varphi) \mathbb{1}_{\mathrm{y}}-\frac{1}{2}\left(q_{\mathrm{x}} \mathbb{1}_{\mathrm{x}}+q_{\mathrm{y}} \mathbb{1}_{\mathrm{y}}\right) \sigma_{\mathrm{x}} \sigma_{\mathrm{y}}: U \in \mathcal{U}^{\varnothing}, \lambda_{\mathrm{x}}, \lambda_{\mathrm{y}}, q_{\mathrm{x}} q_{\mathrm{y}} \in \mathbb{C}\right\} .
\end{aligned}
$$

The field $\varphi$ is evaluated at a point $x \in \Lambda$, and here $\mathbb{1}_{\mathrm{x}}$ represents the Kronecker delta $\mathbb{1}_{\mathrm{x}}(x)=$ $\mathbb{1}_{\mathrm{x}=x}=\delta_{\mathrm{x}, x}$. Given $X \subset \Lambda$, we also define, e.g.,

$$
\mathcal{V}(X)=\left\{V(X)=\sum_{x \in X} V_{x}: V \in \mathcal{V}\right\}
$$

The counterparts of these monomials and spaces for $n=0$ are modified as in [6].

\subsection{Localisation}

Given $X \subset \Lambda$, the localisation operator is a linear map $\operatorname{Loc}_{X}$ which projects $\mathcal{N}$ onto a subspace of polynomials consisting of relevant and marginal monomials summed over $X$, essentially as a Taylor expansion. Since $\operatorname{Loc}_{X}$ preserves $O(n)$ symmetry, in practice it maps into $\mathcal{U}(X)$. The definition and properties of Loc are developed in detail in [14]; it involves parameters which we specify in Section B.1.3. The following example gives the action of $\operatorname{Loc}_{x}$ with these parameters when $X=\{x\}$ is a single point; this is all that is required for the rest of Section 3 .

Example 3.1. For notational simplicity, suppose that the number of field components is $n=1$.

(i) If $r+s$ is even then

$$
\operatorname{Loc}_{x} \varphi_{x}^{r} \varphi_{y}^{s}= \begin{cases}0 & (r+s>6) \\ \varphi_{x}^{r+s} & (r+s=4,6) .\end{cases}
$$

Also, $\operatorname{Loc}_{x} \varphi_{x}^{r}=\varphi_{x}^{r}$ for $r \leq 6$, whereas $\operatorname{Loc}_{x} \varphi_{x}^{r}=0$ for $r>6$.

(ii) Suppose that $p: \Lambda \rightarrow \mathbb{R}$ satisfies $p_{x}=0$ if $|x|>\frac{1}{2} \operatorname{diam}(\Lambda)=\frac{1}{2} L^{N}$ and that, for some $p^{(* *)} \in \mathbb{R}$,

$$
\sum_{x \in \Lambda} p_{x} x_{i}=0, \quad \sum_{x \in \Lambda} p_{x} x_{i} x_{j}=p^{(* *)} \delta_{i, j}, \quad i, j \in\{1,2,3\} .
$$


Then, as in [14, Section 1.5] or [6, (5.29)-(5.30)], with $\tau_{x y}=\frac{1}{2} \varphi_{x} \varphi_{y}$ and $p^{(1)}=\sum_{x} p_{x}$,

$$
\begin{aligned}
\operatorname{Loc}_{x}\left(\sum_{y \in \Lambda} p_{x-y} \tau_{y}\right) & =p^{(1)} \tau_{x}+p^{(* *)}\left(\tau_{\nabla \nabla, x}-\tau_{\Delta, x}\right), \\
\operatorname{Loc}_{x}\left(\sum_{y \in \Lambda} p_{x-y} \tau_{x y}\right) & =p^{(1)} \tau_{x}+p^{(* *)} \tau_{\Delta, x} .
\end{aligned}
$$

\subsection{Definition of the map PT}

In this section, we define the quadratic map $\mathrm{PT}_{j}: \mathcal{U} \rightarrow \mathcal{U}$ ("PT" stands for "perturbation theory"). It is designed in such a way that if $Z_{j}$ is represented perturbatively as $Z_{j} \approx e^{-U_{j}(\Lambda)}$ for a polynomial $U_{j} \in \mathcal{U}$, then the map $Z_{j} \mapsto Z_{j+1}$ can be approximated by the map $U_{j} \mapsto \mathrm{PT}_{j}\left(U_{j}\right)$. This is discussed in detail in [6, Section 2]. We use the notation here for $n \geq 1$; the adaptation to $n=0$ can be found in $[6]$.

Given a $\Lambda \times \Lambda$ matrix $C$, we define a linear operator on sufficiently differentiable complex-valued functions of $\varphi$ by

$$
\mathcal{L}_{C}=\frac{1}{2} \sum_{i=1}^{n} \sum_{u, v \in \Lambda} C_{u, v} \frac{\partial}{\partial \varphi_{u}^{i}} \frac{\partial}{\partial \varphi_{v}^{i}} .
$$

For a polynomial $A$ in the field, $\mathbb{E}_{C} \theta A=e^{\mathcal{L}_{C}} A$, where the exponential is defined by power series expansion which terminates when applied to a polynomial (see [13, Lemma 4.2]). For polynomials $A, B$ in the field, we define

$$
F_{C}(A, B)=e^{\mathcal{L}_{C}}\left(e^{-\mathcal{L}_{C}} A\right)\left(e^{-\mathcal{L}_{C}} B\right)-A B
$$

As in [6, Lemma 5.6], F can be evaluated using

$$
F_{C}\left(A_{x}, B_{y}\right)=\sum_{k=1}^{\operatorname{deg} A \wedge \operatorname{deg} B} \frac{1}{k !} \sum_{i_{1}, \ldots, i_{k}=1}^{n} \sum_{u_{l}, v_{l} \in \Lambda}\left(\prod_{l=1}^{k} C_{u_{l}, v_{l}}\right) \frac{\partial^{k} A_{x}}{\partial \varphi_{u_{1}}^{i_{1}} \cdots \partial \varphi_{u_{k}}^{i_{k}}} \frac{\partial^{k} B_{y}}{\partial \varphi_{v_{1}}^{i_{1}} \cdots \partial \varphi_{v_{k}}^{i_{k}}} .
$$

Let $w_{0}=0$. For $j \geq 1$, and for $C_{i}$ the terms in the covariance decomposition (2.6), let

$$
w_{j}=\sum_{i=1}^{j} C_{i}
$$

The range of $w_{j}$ is the same as that of $C_{j}$, namely $\frac{1}{2} L^{j}$. For $U \in \mathcal{U}$ and $X \subset \Lambda$, we set

$$
W_{j}(U, X)=\frac{1}{2} \sum_{x \in X}\left(1-\operatorname{Loc}_{x}\right) F_{w_{j}}\left(U_{x}, U(\Lambda)\right) .
$$

The map $\mathrm{PT}_{j}: \mathcal{U} \rightarrow \mathcal{U}$ is then defined by

$$
\mathrm{PT}_{j}(U)=e^{\mathcal{L}_{C_{j+1}}} U-P_{j}(U)
$$


with

$$
P_{x, j}(U)=\operatorname{Loc}_{x}\left(e^{\mathcal{L}_{C_{j+1}}} W_{j}(U, x)+\frac{1}{2} F_{C_{j+1}}\left(e^{\mathcal{L}_{C_{j+1}}} U_{x}, e^{\left.\mathcal{L}_{C_{j+1}} U(\Lambda)\right)}\right)\right.
$$

By translation invariance, $P_{x, j}(U)$ defines a local polynomial with coefficients independent of $x$. According to [6, Lemma 5.5], an equivalent alternate formula for $P_{x, j}(U)$ is

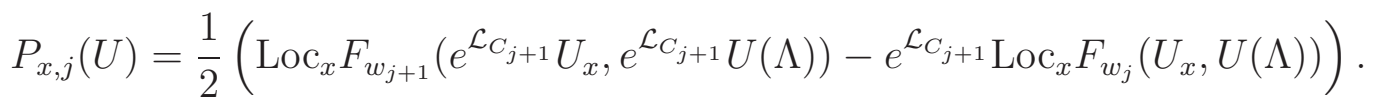

\subsection{Linear term}

Throughout Sections 3.3-3.5, we study only on the bulk, and return to observables in Section 4.2.

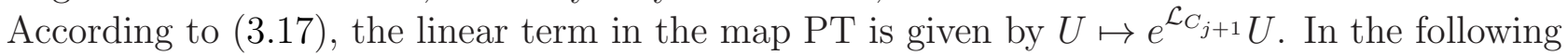
lemma, we compute this linear map, for $U \in \mathcal{U}^{\varnothing}$ and for an arbitrary covariance $C$. The matrix is with respect to the representation of $U=a \tau^{3}+g \tau^{2}+\nu \tau+y \tau_{\nabla \nabla}+z \tau_{\Delta}+u \in \mathcal{U}^{\varnothing}$ as

$$
U=(u, \nu, g, a, y, z) \text {. }
$$

Lemma 3.2. Let $n \geq 0$. The linear map $e^{\mathcal{L}_{C}}$ on $\mathcal{U}$ has matrix representation, with $c=C_{0,0}$ and $c_{\Delta}=\Delta C_{0,0}$,

$$
e^{\mathcal{L}_{C}}=\left(\begin{array}{cccccc}
1 & \frac{1}{2} n c & \frac{1}{4} n(n+2) c^{2} & \frac{3}{32} n(n+2)(n+4) c^{3} & -\frac{1}{2} n c_{\Delta} & -\frac{1}{2} n c_{\Delta} \\
0 & 1 & (n+2) c & \frac{3}{4}(n+4)(n+2) c^{2} & 0 & 0 \\
0 & 0 & 1 & \frac{3}{2}(n+4) c & 0 & 0 \\
0 & 0 & 0 & 1 & 0 & 0 \\
0 & 0 & 0 & 0 & 0 & 0 \\
0 & 0 & 0 & 0 & 0 & 1
\end{array}\right) .
$$

Proof. The exponential of $\mathcal{L}=\mathcal{L}_{C}$ is defined by expansion in Taylor series, which gives, for $U \in \mathcal{U}$,

$$
e^{\mathcal{L}} U=\left(1+\mathcal{L}+\frac{1}{2 !} \mathcal{L}^{2}+\frac{1}{3 !} \mathcal{L}^{3}\right) U
$$

Suppose first that $n \geq 1$. Differentiation gives

$$
\begin{aligned}
\mathcal{L}|\varphi|^{2}=n c, \quad \mathcal{L}|\varphi|^{4} & =\frac{1}{2} 4(n+2) c|\varphi|^{2}, \quad \mathcal{L}|\varphi|^{6}=\frac{1}{2} 6(n+4) c|\varphi|^{4}, \\
\mathcal{L}(\varphi \cdot \Delta \varphi) & =\frac{1}{2} \sum_{|e|_{1}=1} \mathcal{L}\left(\nabla^{e} \varphi \cdot \nabla^{e} \varphi\right)=-n \Delta C_{0,0},
\end{aligned}
$$

from which the desired formula can be obtained after some algebra. Similar computations in the supersymmetric setting give the result for $n=0$, as in [6].

\subsection{Dimensionless form of the perturbative flow}

We define $D=\{0,1,2,3, \nabla \nabla, \Delta\}$ as a set whose elements are representatives of the monomials $1, \tau, \tau^{2}, \tau^{3}, \tau_{\nabla \nabla}, \tau_{\Delta}$. We write these monomials as $M^{i}$ for $i \in D$. The dimension of $i \in\{0,1,2,3\}$ is defined to be $[i]=i$, and $[\nabla \nabla]=[\Delta]=3$. 
We rewrite the coupling constants as

$$
\gamma_{0}=u, \quad \gamma_{1}=\nu, \quad \gamma_{2}=g, \quad \gamma_{3}=a, \quad \gamma_{\nabla \nabla}=y, \quad \gamma_{\Delta}=z
$$

By (3.17), the perturbative flow equations, which express the coupling constants of PT $(U)$ in terms of those of $U$, have the form

$$
\gamma_{i, \mathrm{pt}}=\sum_{p \in D} \alpha_{i}^{p} \gamma_{p}-\sum_{p, q \in D} \alpha_{i}^{p q} \gamma_{p} \gamma_{q}
$$

The linear coefficients $\alpha_{i}^{p}$ are matrix elements of (3.21). The quadratic coefficients $\alpha_{i}^{p q}$ are our main concern in the rest of this section. They can be computed exactly as in $[3,5]$, but mostly we do not need exact values here. The following lemma obtains estimates much more efficiently than those obtained from exact formulas in $[3,6]$. Recall that $\vartheta_{j}$ is defined in $(2.9)$.

Lemma 3.3. For $i, p, q \in D$, the coefficients $\alpha_{i}^{p}, \alpha_{i}^{p q}$ in (3.26) obey the estimates

$$
\begin{aligned}
\alpha_{i}^{i} & =1, \\
\alpha_{0}^{p} & =O\left(\vartheta_{j} L^{-j[p]}\right) \quad(p \neq 0), \\
\alpha_{1}^{p} & =O\left(\vartheta_{j} L^{-j([p]-1)}\right) \quad(p=2,3), \\
\alpha_{2}^{3} & =O\left(\vartheta_{j} L^{-j(3-2)}\right), \\
\alpha_{i}^{p q} & =O L\left(\vartheta_{j} L^{j(3+[i]-[p]-[q])}\right),
\end{aligned}
$$

and all $\alpha_{i}^{p}$ not listed above are equal to zero.

Proof. For the linear terms, we can read off the coefficients from Lemma 3.2, and the bound follows from the estimate $(2.8)$ on the covariance.

For the quadratic terms, we use the $T_{0}(\ell)$-seminorm (defined in [3, Section 2.3] for $n \geq 1$ and in $[5$, Section 6.3] for $n=0)$ with parameter

$$
\ell_{j}=\ell_{0} L^{-j[\varphi]}=\ell_{0} L^{-j / 2}
$$

with $\ell_{0}$ a (large) $L$-dependent constant. If $B$ is a $j$-block, then a calculation gives

$$
\left\|M^{r}(B)\right\|_{T_{0}(\ell)} \asymp_{L} L^{j(3-[r])}
$$

where the notation $\asymp_{L}$ indicates upper and lower bounds with constants that may depend on $L$. We apply [15, Proposition 4.10] and (3.33) to see that

$$
\left\|P\left(M^{p}(B), M^{q}(B)\right)\right\|_{T_{0}(\ell)}=O_{L}(\vartheta) L^{j(3-[p])} L^{j(3-[q])} .
$$

Since, by definition,

$$
P\left(M^{p}(B), M^{q}(B)\right)=\sum_{i \in D} \alpha_{i}^{p q} M^{i}(B),
$$

it follows from the fact that $\left\|\alpha_{i}^{p q} M^{i}(B)\right\|_{T_{0}(\ell)} \leq\left\|\sum_{i \in D} \alpha_{i}^{p q} M^{i}(B)\right\|_{T_{0}(\ell)}($ as in $[15,(3.4)])$ that

$$
\left\|\alpha_{i}^{p q} M^{i}(B)\right\|_{T_{0}(\ell)} \leq O_{L}(\vartheta) L^{j(6-([p]+[q]))}
$$

so that

$$
\left|\alpha_{i}^{p q}\right| \leq O_{L}(\vartheta) L^{j(6-([p]+[q]))} L^{-j(3-[i])}=O_{L}(\vartheta) L^{j(3+[i]-[p]-[q])} .
$$

This completes the proof. 
We now rescale to dimensionless variables, as follows. Let

$$
\hat{\gamma}_{i}=L^{(3-[i]) j} \gamma_{i}, \quad \gamma_{i}=L^{-(3-[i]) j} \hat{\gamma}_{i} .
$$

We define dimensionless coefficients, with all $\hat{\alpha}_{i}^{p}, \hat{\alpha}_{i}^{p q}$ bounded by $\vartheta\left(\right.$ except $\hat{\alpha}_{i}^{i}=1$ ), by

$$
\left.\hat{\alpha}_{i}^{p}=L^{j([p]-[i])} \alpha_{i}^{p}, \quad \hat{\alpha}_{i}^{p q}=L^{j(-3-[i]+[p]+[q])}\right) \alpha_{i}^{p q} .
$$

Then we can rewrite the original perturbative flow equations (3.26) in dimensionless form as

$$
\hat{\gamma}_{i, \mathrm{pt}}=L^{3-[i]}\left(\sum_{p \in D} \hat{\alpha}_{i}^{p} \hat{\gamma}_{p}-\sum_{p, q \in D} \hat{\alpha}_{i}^{p q} \hat{\gamma}_{p} \hat{\gamma}_{q}\right) \quad(i \in D) .
$$

\subsection{Change of variables}

In this section, we make a change of variables and transform the dimensionless perturbative flow equations (3.40) into a triangular form. This is achieved in Proposition 3.5. A related transformation was used in [6, Section 4.2] in a more ad hoc manner. Here we present the transformation in a systematic way.

Let $U \in \mathcal{U}^{\varnothing}$. We write $F=F_{w}, F_{+}=F_{w+C}$, and $\mathcal{L}_{+}=\mathcal{L}_{w+C}$. By (3.19), the quadratic term in $\mathrm{PT}(U)$ is

$$
P_{x}(U)=\frac{1}{2}\left(\operatorname{Loc}_{x} F_{+}\left(e^{\mathcal{L}_{+}} U_{x} ; e^{\mathcal{L}_{+}} U(\Lambda)\right)-e^{\mathcal{L}_{+}} \operatorname{Loc}_{x} F\left(U_{x} ; U(\Lambda)\right)\right) .
$$

Let $Q(U, U)=\frac{1}{2} \operatorname{Loc}_{x} F\left(U_{x} ; U(\Lambda)\right)$. There are coefficients $\kappa_{i}^{p q}(i, p, q \in D)$ such that

$$
Q\left(M_{x}^{p}, M^{q}(\Lambda)\right)=\sum_{i \in D} \kappa_{i}^{p q} M_{x}^{i} .
$$

Rescaled versions of the coefficients are defined, as in (3.39), by

$$
\hat{\kappa}_{i}^{p q}=L^{j(-3-[i]+[p]+[q])} \kappa_{i}^{p q} .
$$

The coefficients $\hat{\kappa}_{i}^{p q}$ may be bounded or unbounded in the scale $j$, and we define

$$
S^{\mathrm{bd}}=\left\{(i, p, q): \hat{\kappa}_{i}^{p q}=O(1)\right\},
$$

and, for $U=\sum_{i \in D} \gamma_{i} M^{i}$, set

$$
Q^{\mathrm{bd}}(U, U)=\sum_{(i, p, q) \in S^{\mathrm{bd}}} \kappa_{i}^{p q} \gamma_{p} \gamma_{q} M_{x}^{i}, \quad Q^{\mathrm{div}}(U, U)=Q(U, U)-Q^{\mathrm{bd}}(U, U) .
$$

Thus we have divided $Q$ into its bounded and unbounded terms as $Q=Q^{\mathrm{bd}}+Q^{\mathrm{div}}$, so

$$
\begin{aligned}
P_{x}(U)= & Q_{+}\left(e^{\mathcal{L}_{+}} U ; e^{\mathcal{L}_{+}} U\right)-e^{\mathcal{L}_{+}} Q(U, U) \\
= & {\left[Q_{+}^{\mathrm{div}}\left(e^{\mathcal{L}_{+}} U ; e^{\mathcal{L}_{+}} U\right)-e^{\mathcal{L}_{+}} Q^{\mathrm{div}}(U, U)\right] } \\
& +\left[Q_{+}^{\mathrm{bd}}\left(e^{\mathcal{L}_{+}} U ; e^{\mathcal{L}_{+}} U\right)-e^{\mathcal{L}_{+}} Q^{\mathrm{bd}}(U, U)\right] .
\end{aligned}
$$


We write the map PT as $\varphi_{\mathrm{pt}}$, set $U_{\mathrm{pt}}=\varphi_{\mathrm{pt}}(U)$, and rewrite the equation $U_{\mathrm{pt}}=e^{\mathcal{L}+} U-P$ as

$$
\varphi_{\mathrm{pt}}(U)+Q_{+}^{\mathrm{bd}}\left(e^{\mathcal{L}_{+}} U ; e^{\mathcal{L}_{+}} U\right)=e^{\mathcal{L}_{+}}\left(U+Q^{\mathrm{bd}}(U, U)\right)-\left[Q_{+}^{\operatorname{div}}\left(e^{\mathcal{L}_{+}} U ; e^{\mathcal{L}_{+}} U\right)-e^{\mathcal{L}_{+}} Q^{\operatorname{div}}(U, U)\right] .
$$

Next, we define a transformation $T: \mathcal{U} \rightarrow \mathcal{U}$ by

$$
T(U)=U+Q^{\mathrm{bd}}(U, U) .
$$

Note that $T$ is equal to the identity map plus a quadratic part. It follows from (3.47) that

$$
\begin{aligned}
& \varphi_{\mathrm{pt}}(U)+Q_{+}^{\mathrm{bd}}\left(\varphi_{\mathrm{pt}}(U) ; \varphi_{\mathrm{pt}}(U)\right) \\
& \quad=e^{\mathcal{L}_{+}}\left(U+Q^{\mathrm{bd}}(U, U)\right)-\left[Q_{+}^{\mathrm{div}}\left(e^{\mathcal{L}_{+}} T(U) U ; e^{\mathcal{L}_{+}} T(U)\right)-e^{\mathcal{L}_{+}} Q^{\operatorname{div}}(T(U), T(U))\right]+O\left(U^{3}\right),
\end{aligned}
$$

and hence

$$
\left(T_{+} \circ \varphi_{\mathrm{pt}} \circ T^{-1}\right)(U)=e^{\mathcal{L}_{+}} U-\left[Q_{+}^{\mathrm{div}}\left(e^{\mathcal{L}_{+}} U ; e^{\mathcal{L}_{+}} U\right)-e^{\mathcal{L}_{+}} Q^{\operatorname{div}}(U, U)\right]+O\left(U^{3}\right) .
$$

The approximate flow is defined by dropping the error term in the above, which yields

$$
\bar{U}_{+}=e^{\mathcal{L}_{+}} \bar{U}-\left[Q_{+}^{\operatorname{div}}\left(e^{\mathcal{L}_{+}} \bar{U} ; e^{\mathcal{L}_{+}} \bar{U}\right)-e^{\mathcal{L}_{+}} Q^{\operatorname{div}}(\bar{U}, \bar{U})\right]
$$

The following lemma identifies several coefficients whose indices belong to $S^{\text {bd }}$. The proof of the lemma shows that many of the options in (3.55) are in fact zero, but since we do not need to know they are zero, we state the weaker bounds for simplicity. It is not necessary to transform the variables $u, y$ so we omit them from the following discussion.

Lemma 3.4. For $i, p, q \in\{1,2,3, \Delta\}$, the coefficients obey

$$
\begin{array}{rlrl}
\hat{\kappa}_{i}^{p q} & =O(1) & & (p+q<3+i, i, p, q \in\{1,2,3\}), \\
\hat{\kappa}_{1}^{13}=\hat{\kappa}_{1}^{31}=O(1), & & \\
\hat{\kappa}_{\Delta}^{p q} & =O(1) & & (p, q \in\{1,2,3\} \operatorname{except}(p, q)=(3,3)), \\
\hat{\kappa}_{i}^{\Delta p}=\hat{\kappa}_{i}^{p \Delta}=O(1) & & (i, p \in\{1,2,3, \Delta\}) .
\end{array}
$$

Before proving the lemma, we discuss its important consequence that the approximate flow is triangular.

Proposition 3.5. The approximate flow has the following form, with all coefficients $\bar{\beta}=O(\vartheta)$ :

$$
\begin{aligned}
\bar{\gamma}_{3,+} & =\bar{\gamma}_{3}-\bar{\beta}_{3}^{33} \bar{\gamma}_{3}^{2} \\
\bar{\gamma}_{\Delta,+} & =\bar{\gamma}_{\Delta}-\bar{\beta}_{\Delta}^{33} \bar{\gamma}_{3}^{2}, \\
\bar{\gamma}_{2,+} & =L\left(\bar{\gamma}_{2}+\bar{\beta}_{2}^{3} \bar{\gamma}_{3}-\bar{\beta}_{2}^{23} \bar{\gamma}_{2} \bar{\gamma}_{3}-\bar{\beta}_{2}^{33} \bar{\gamma}_{3}^{2}\right) \\
\bar{\gamma}_{1,+} & =L^{2}\left(\bar{\gamma}_{1}+\bar{\beta}_{1}^{2} \bar{\gamma}_{2}+\bar{\beta}_{1}^{3} \bar{\gamma}_{3}-\bar{\beta}_{1}^{22} \bar{\gamma}_{2}^{2}-\bar{\beta}_{1}^{23} \bar{\gamma}_{2} \bar{\gamma}_{3}-\bar{\beta}_{1}^{33} \bar{\gamma}_{3}^{2}\right)
\end{aligned}
$$

Proof assuming Lemma 3.4. The linear terms on the right-hand sides of (3.56)-(3.59) have the desired form by Lemma 3.3, so we only need to study the quadratic terms. By Lemma 3.3, the quadratic coefficients are all $O(\vartheta)$.

It follows from (3.52) that there are no $\bar{\gamma}_{1}$ or $\bar{\gamma}_{2}$ terms in the $\bar{\gamma}_{3}$ equation, that there are no $\bar{\gamma}_{1}$ terms in the $\bar{\gamma}_{2}$ equation, and that there is no $\bar{\gamma}_{1} \bar{\gamma}_{2}$ or $\bar{\gamma}_{1} \bar{\gamma}_{1}$ term in the $\bar{\gamma}_{1}$ equation.

It follows from (3.53) that there is no $\bar{\gamma}_{1} \bar{\gamma}_{3}$ term in the $\bar{\gamma}_{1}$ equation.

It follows from (3.54) that the $\bar{\gamma}_{\Delta}$ equation can only have a $\bar{\gamma}_{3}^{2}$ term, among $\bar{\gamma}_{1}, \bar{\gamma}_{2}, \bar{\gamma}_{3}$.

It follows from $(3.55)$ that no $\bar{\gamma}_{\Delta}$ terms occur in any equation. 
Concerning the coefficients $\bar{\beta}$, by Lemma 3.2 the linear ones are

$$
\begin{gathered}
\bar{\beta}_{1, j}^{2}=(n+2) L^{j} C_{j+1 ; 0,0}, \quad \bar{\beta}_{1, j}^{3}=\frac{3}{4}(n+4)(n+2) L^{2 j} C_{j+1 ; 0,0}^{2}, \\
\bar{\beta}_{2, j}^{3}=\frac{3}{2}(n+4) L^{j} C_{j+1 ; 0,0} .
\end{gathered}
$$

For the quadratic coefficients, we extend (3.15) by defining, for integers $j \geq 0$ and $k \geq 1$,

$$
w_{j}=\sum_{k=1}^{j} C_{k}, \quad w_{j}^{(k)}=\sum_{x \in \mathbb{Z}^{d}} w_{j ; 0 x}^{k}, \quad w_{j}^{(k, * *)}=\sum_{x \in \mathbb{Z}^{d}} x_{1}^{2} w_{j ; 0 x}^{k} .
$$

Bounds on these quantities are given in Lemma A.2. Two marginal coefficients play a specific role, which can be computed directly (including the case $n=0$ ) as

$$
\bar{\beta}_{3}^{33}=(18 n+132) \delta\left[w^{(3)}\right], \quad \bar{\beta}_{2}^{23}=(12 n+48) \delta\left[w^{(3)}\right],
$$

where we write in general $\delta[f]=f_{j+1}-f_{j}$. For later use, we define $p_{2}$ as the ratio

$$
p_{2}=\frac{\bar{\beta}_{2}^{23}}{\bar{\beta}_{3}^{33}}=\frac{2(n+4)}{3 n+22} \text {. }
$$

By Proposition 3.5, $\delta\left[w^{(3)}\right]$ is bounded, but in fact this results from a difference of unbounded terms. Although we do not need it, explicit computation also gives

$$
\bar{\beta}_{1}^{22}=(2 n+4) \delta\left[w^{(3)}\right], \quad \bar{\beta}_{\Delta}^{33}=-\frac{9}{4}(n+2)(n+4) \delta\left[w^{(5, * *)}\right]+\text { bounded } .
$$

Proof of Lemma 3.4. For notational simplicity, suppose that the number of field components is $n=1$. Recall from (3.43) that $\hat{\kappa}_{i}^{p q}=L^{j(-3-[i]+[p]+[q])} \kappa_{i}^{p q}$, where $\kappa_{i}^{p q}$ is the coefficient appearing in

$$
Q\left(M_{x}^{p}, M^{q}(\Lambda)\right)=\sum_{y} \frac{1}{2} \operatorname{Loc}_{x} F\left(M_{x}^{p}, M_{y}^{q}\right)=\sum_{i \in D} \kappa_{i}^{p q} M_{x}^{i} .
$$

Proof of (3.52). Suppose first that $p, q \in\{1,2,3\}$ and that $p+q-i<3$. By the formula for $F$ in (3.14), there are $c_{p, q, k}$ such that

$$
\operatorname{Loc}_{x} F\left(\varphi_{x}^{2 p}, \varphi_{y}^{2 q}\right)=\sum_{k=1}^{2 p \wedge 2 q} c_{p, q, k} w_{x y}^{k} \operatorname{Loc}_{x} \varphi_{x}^{2 p-k} \varphi_{y}^{2 q-k}
$$

By Example 3.1, if $p+q-k \geq 2$ then

$$
\operatorname{Loc}_{x} \varphi_{x}^{2 p-k} \varphi_{y}^{2 q-k}=\mathbb{1}_{p+q-k \leq 3} \varphi_{x}^{2(p+q-k)},
$$

and hence, for $i=p+q-k$ (which entails $k<3$ by hypothesis),

$$
\hat{\kappa}_{i}^{p q} \propto L^{j(-3-i+p+q)} w_{j}^{(k)}=O(1)
$$


since $w_{j}^{(k)} \leq O\left(L^{j(3-k)}\right)$ by Lemma A.2. If instead $p+q-k=1$ then it follows from Example 3.1 that

$$
\operatorname{Loc}_{x} \sum_{y} \varphi_{x}^{2 p-k} w_{x y}^{k} \varphi_{y}^{2 q-k}=\varphi_{x}^{2} w^{(k)}+\mathbb{1}_{2 q-k>0} \varphi_{\Delta, x}^{2} w^{(k, * *)}
$$

where $\varphi_{\Delta, x}^{2}$ represents a linear combination of $\tau_{\Delta, x}$ and $\tau_{\nabla \nabla, x}$. The first term in (3.70) yields $\hat{\kappa}_{1}^{p q}$, which is $O(1)$ as in $(3.69)$.

Proof of (3.54). The second term in (3.70) gives rise to $\hat{\kappa}_{\Delta}^{p q}$ and $\hat{\kappa}_{\nabla \nabla}^{p q}$, and the relation $p+q=k+1$ implies that $k<5$ if we exclude $(p, q)=(3,3)$. Therefore, by Example 3.1 and by Lemma A.2, these contributions are bounded above by

$$
L^{j(-3-3+p+q)} O\left(L^{j(5-k)}\right)=L^{j(k-5)} O\left(L^{j(5-k)}\right)=O(1),
$$

which proves $(3.54)$. (In (3.70), the marginal term $w^{(5, * *)}$ arises for $\hat{\kappa}_{\Delta}^{33}$.)

Proof of (3.53). The coefficient $\hat{\kappa}_{1}^{13}$ violates the assumption $p+q-i<3$ of (3.52). However, this coefficient is zero because $F\left(\varphi_{x}^{2}, \varphi_{y}^{6}\right)$ and $F\left(\varphi_{x}^{6}, \varphi_{y}^{2}\right)$ contribute no $\varphi^{2}$ term because the maximal number of field derivatives in (3.14) is two on each factor, and this leaves $\varphi^{4}$, not $\varphi^{2}$.

Proof of (3.55). There are three cases: $F\left(\tau_{\Delta}, \tau_{\Delta}\right), F\left(\tau_{\Delta}, \varphi^{2 q}\right)$, and $F\left(\varphi^{2 p}, \tau_{\Delta}\right)$. For the first,

$$
\begin{aligned}
\operatorname{Loc}_{x} \sum_{y} F\left(\Delta \varphi_{x}^{2}, \Delta \varphi_{y}^{2}\right) & =\operatorname{Loc}_{x} \sum_{y} \Delta_{x} \Delta_{y} F\left(\varphi_{x}^{2}, \varphi_{y}^{2}\right) \\
& =\sum_{y} \Delta_{x} \Delta_{y} \operatorname{Loc}_{x}\left(c_{1} \varphi_{x} w_{x y} \varphi_{y}+c_{2} w_{x y}^{2}\right) \\
& =\Delta_{x} \sum_{y} \Delta_{y} w_{x y}\left(c_{1} \varphi_{x}^{2}+c_{1}^{\prime}(y-x)^{2} \varphi_{\Delta, x}^{2}\right)+\Delta_{x} \sum_{y} \Delta_{y} c_{2} w_{x y}^{2} \\
& =0
\end{aligned}
$$

with the last equality a consequence of summation by parts. For the second case,

$$
\begin{aligned}
\operatorname{Loc}_{x} \sum_{y} F\left(\Delta_{x} \varphi_{x}^{2}, \varphi_{y}^{2 q}\right) & =\operatorname{Loc}_{x} \sum_{y} \Delta_{x} F\left(\varphi_{x}^{2}, \varphi_{y}^{2 q}\right) \\
& =\sum_{y} \Delta_{x} \operatorname{Loc}_{x}\left(c_{1} \varphi_{x} w_{x y} \varphi_{y}^{2 q-1}+c_{2} w_{x y}^{2} \varphi_{y}^{2 q-2}\right) .
\end{aligned}
$$

If $q=3$ then $\operatorname{Loc}_{x}$ simply replaces $y$ by $x$ and the result is zero because $\sum_{y} \Delta_{x} w_{x y}^{m}=\sum_{y} \Delta_{y} w_{x y}^{m}=0$. If $q=2$ then $\operatorname{Loc}_{x}$ replaces $\varphi_{y}^{3}$ by $\varphi_{x}^{3}$ in the first term, and replaces $\varphi_{y}^{2}$ by by $\varphi_{x}^{2}+(y-x)^{2} \Delta \varphi_{x}^{2}$ in the second term. The overall result is again zero. If $q=1$ then the $w^{2}$ term vanishes due to the Laplacian, and the other term becomes $\varphi_{x}^{2}(\Delta w)^{(1)}+\varphi_{\Delta}^{2}(\Delta w)^{(1, * *)}$. The first of these terms is zero, and the second yields a coefficient $\hat{\kappa}_{\Delta}^{\Delta, 2}$ which by Lemma A.2 is at most

$$
L^{-j(3+3-3-1)}(\Delta w)^{(1, * *)}=L^{-j(3+3-3-1)} O\left(L^{j 2}\right)=O(1) .
$$

Finally, for the third case,

$$
\operatorname{Loc}_{x} \sum_{y} F\left(\varphi_{x}^{2 p}, \Delta \varphi_{y}^{2}\right)=\sum_{y} \Delta_{y} \operatorname{Loc}_{x}\left(c_{1} \varphi_{x}^{2 p-1} w_{x y} \varphi_{y}+c_{2} \varphi_{x}^{2 p-2} w_{x y}^{2}\right) .
$$


The last term vanishes due to the Laplacian. For $p \geq 2$ the effect of $\operatorname{Loc}_{x}$ on the first term is the replacement of $\varphi_{y}$ by $\varphi_{x}$ and the result vanishes due to the Laplacian. For $p=1$ the first term is

$$
\sum_{y} \Delta_{y} w_{x y}\left(c_{1} \varphi_{x}^{2}+c_{1}^{\prime}(y-x)^{2} \varphi_{\Delta}^{2}\right)
$$

and again this vanishes by summation by parts.

\section{Analysis of the RG flow}

In this section, we analyse the RG flow in preparation for the proof of Theorems 1.1-1.2. The bulk flow is discussed in Section 4.1, and the observable flow in Section 4.2.

\subsection{Analysis of bulk flow}

In this section, we analyse the approximate flow of Proposition 3.5, modified by inclusion of remainder terms produced by Theorem 2.1. We write the variables in the modified approximate flow as $\mu$ rather than $\bar{\gamma}$. By Proposition 3.5, (3.64) and Theorem 2.1, the modified approximate flow is

$$
\begin{aligned}
\mu_{3,+} & =\mu_{3}-\bar{\beta}_{3}^{33} \mu_{3}^{2}+e_{3} \\
\mu_{\Delta,+} & =\mu_{\Delta}-\bar{\beta}_{\Delta}^{33} \mu_{3}^{2}+e_{\Delta}, \\
\mu_{2,+} & =L\left(\mu_{2}\left(1-p_{2} \bar{\beta}_{3}^{33} \mu_{3}\right)+\bar{\beta}_{2}^{3} \mu_{3}-\bar{\beta}_{2}^{33} \mu_{3}^{2}\right)+e_{2}, \\
\mu_{1,+} & =L^{2}\left(\mu_{1}-\rho_{1}\right)
\end{aligned}
$$

with

$$
\rho_{1}=-\bar{\beta}_{1}^{2} \mu_{2}-\bar{\beta}_{1}^{3} \mu_{3}+\bar{\beta}_{1}^{22} \mu_{2}^{2}+\bar{\beta}_{1}^{23} \mu_{2} \mu_{3}+\bar{\beta}_{1}^{33} \mu_{3}^{2}-e_{1},
$$

with all coefficients $\bar{\beta}=O(\vartheta)$, and with $e_{*}=O\left(\vartheta \mu_{3}^{3}\right)$. The approximate flow is the special case with $e_{*}=0$.

A solution to the $\mu_{3}$ flow is given in [8, Section 6.1.1], which in our present setting yields the statements in the following proposition. Recall from (3.63) that $\bar{\beta}_{3}^{33}=\mathrm{b} \delta\left[w^{(3)}\right]$ with $\mathrm{b}=18 n+132$. The $L$-dependent constant $\lambda_{3}$ in Proposition 4.1(i) arises in Lemma A.3.

Proposition 4.1. Let $m^{2} \in[0, \delta)$ and $\mu_{3,0} \in(0, \delta)$.

(i) If $m^{2}=0$ then $\mu_{3, j}(0) \sim 1 /\left(\mathrm{b} \lambda_{3} j\right) \rightarrow 0$ as $j \rightarrow \infty$. For $m^{2}>0$, the limit $\mu_{3, \infty}\left(m^{2}\right)=$ $\lim _{j \rightarrow \infty} \mu_{3, j}\left(m^{2}\right)>0$ exists, is continuous in $m^{2}$, and obeys $\mu_{3, \infty}\left(m^{2}\right) \sim 1 /\left(\mathrm{b} w_{\infty}^{(3)}\left(m^{2}\right)\right)$ as $m^{2} \downarrow 0$.

(ii) The sequence $\mu_{3, j}$ obeys $\mu_{3, j}=O\left(\mu_{3,0}\right), \mu_{3, j+1}=\mu_{3, j}\left(1+O\left(\mu_{3,0}\right)\right), \vartheta_{j}\left(m^{2}\right) \mu_{3, j}\left(m^{2}\right) \leq O\left(\mu_{3, j}(0)\right)$, and

$$
\sum_{l=j}^{\infty} \vartheta_{l} \mu_{3, l}^{p} \leq O\left(\vartheta_{j} \mu_{3, j}^{p-1}\right) \quad(p>1)
$$


Lemma 4.2. If $\left|f_{j}\right| \leq O\left(A^{-j}\right)$ for some $A>1$, and if $p>0$, then

$$
\sum_{j=0}^{\infty} f_{j} \mu_{3, j}^{p}=\mu_{3,0}^{p} \sum_{j=0}^{\infty} f_{j}+O\left(\mu_{3,0}^{p+1}\right) .
$$

Proof. We apply $[8,(13.6 .12)]$ with $\psi(t)=p t^{p-1}$, and then use (4.6) and the fact that $\beta_{3, l}^{33}=O\left(\vartheta_{l}\right)$ by Proposition 3.5, to obtain

$$
\begin{aligned}
\mu_{3,0}^{p}-\mu_{3, j}^{p} & =p \sum_{l=0}^{j-1}\left(\beta_{3, l}^{33} \mu_{3, l}^{2}-e_{3, l}\right) \mu_{3, l}^{p-1}+O\left(\mu_{3,0}^{p+1}\right) \\
& =p \sum_{l=0}^{j-1} \beta_{3, l}^{33} \mu_{3, l}^{p+1}+O\left(\mu_{3,0}^{p+1}\right) .
\end{aligned}
$$

Therefore, after interchanging sums and using $\mu_{3, l}=O\left(\mu_{3,0}\right)$, we obtain

$$
\sum_{j=0}^{\infty} f_{j}\left(\mu_{3,0}^{p}-\mu_{3, j}^{p}\right)=\sum_{l=0}^{\infty} \beta_{3, l}^{33} \mu_{3, l}^{p+1} O\left(f_{l}\right)+O\left(\mu_{3,0}^{p+1}\right)=O\left(\mu_{3,0}^{p+1}\right),
$$

as required.

The other three equations can be solved backwards with zero final condition. For $\mu_{\Delta}$, this gives

$$
\mu_{\Delta, j}=\sum_{l=j}^{\infty}\left(\bar{\beta}_{\Delta, l}^{33} \mu_{3, l}^{2}-e_{\Delta, l}\right)
$$

which converges by (4.6). For $\mu_{2}$, we write the equation backwards and solve with zero final condition to get

$$
\mu_{2, j}=\sum_{l=j}^{\infty} L^{-(l-j)} \pi_{j, l}^{-1}\left(-\bar{\beta}_{2, l}^{3} \mu_{3, l}+\bar{\beta}_{2, l}^{33} \mu_{3, l}^{2}-e_{2, l}\right)
$$

with

$$
\pi_{i, j}=\prod_{k=i}^{j}\left(1-p_{2} \bar{\beta}_{3, k}^{33} \mu_{3, k}\right)
$$

By [8, Lemma 6.1.6],

$$
\pi_{i, j}=\left(\frac{\mu_{3, j+1}}{\mu_{3, i}}\right)^{p_{2}}\left(c_{i}+O\left(\vartheta_{j} \mu_{3, j}\right)\right) \quad \text { with } \quad c_{i}=1+O\left(\vartheta_{i} \mu_{3, i}\right) .
$$

Finally,

$$
\mu_{1, j}=\sum_{l=j}^{\infty} L^{-2(l-j)} \rho_{1, l}
$$

The powers of $L$ give exponential convergence of the sums in (4.11) and (4.14). 


\subsection{Analysis of observable flow}

The bulk flow has been constructed above, with the critical initial conditions given by Theorem 2.1. We now construct the observable flow in terms of the bulk flow.

\subsubsection{Flow of $\lambda$}

The perturbative flow of $\lambda$ is as in [35, Proposition 3.2] (or [6, (3.34)] for $n=0$ ). Namely, for $x=\mathrm{x}, \mathrm{y}$,

$$
\lambda_{x, \mathrm{pt}}= \begin{cases}\left(1-\delta\left[\nu w^{(1)}\right]\right) \lambda_{x} & \left(j+1<j_{\mathrm{xy}}\right) \\ \lambda & \left(j+1 \geq j_{\mathrm{xy}}\right) .\end{cases}
$$

Here $j$ refers to the scale of the input $V, w^{(1)}$ is given by (3.62) and is $O\left(L^{2 j}\right)$ by Lemma A.2, and

$$
\delta_{j}\left[\nu w^{(1)}\right]=\nu_{j}^{+} w_{j+1}^{(1)}-\nu_{j} w_{j}^{(1)}
$$

with $\nu_{j}^{+}$defined to be the first order part of (4.3), namely

$$
\nu_{j}^{+}=\nu_{j}+\bar{\beta}_{1, j}^{2} L^{-j} g_{j}+\bar{\beta}_{1, j}^{3} L^{-2 j} a_{j}
$$

Here $\nu_{j}^{+}$contains an $a$-dependent term which is absent absent in $[6,(3.24)]$ where there was no $|\varphi|^{6}$ term.

Note that the perturbative flow of $\lambda$ stops one scale prior to the coalescence scale. Since $\mathrm{x}, \mathrm{y}$ are points in the torus $\Lambda_{N}$, we always have $N \geq j_{\text {xy }}$, so the perturbative flow stops before reaching scale $N$. By Theorem 2.2, the nonperturbative flow also stops prior to the coalescence scale and is given by

$$
\lambda_{x, j+1}=\left(1-\delta_{j}\left[\nu w^{(1)}\right]\right) \lambda_{x, j}+R_{j+1}^{\lambda_{x}} \quad\left(j+1<j_{\mathrm{xy}}\right),
$$

with, for some $M=M(L)$,

$$
\left|R_{j+1}^{\lambda_{x}}\right| \leq M \vartheta_{j} a_{j}^{2} \mathbb{1}_{j+1<j_{x y}}
$$

Let

$$
f_{j}=1-\delta_{j}\left[\nu w^{(1)}\right], \quad \Pi_{j}=\prod_{k=0}^{j} f_{k} .
$$

Lemma 4.3. There exists $\alpha=1+O\left(a_{0}\right)$, independent of $j$, such that

$$
\Pi_{j}=\alpha\left(1+O\left(\vartheta_{j} a_{j}\right)\right) .
$$

Proof. Let $\delta_{j}=\delta_{j}\left[\nu w^{(1)}\right], \delta_{j}^{\prime}=\nu_{j+1} w_{j+1}^{(1)}-\nu_{j} w_{j}^{(1)}$, and $\Delta_{j}=\left(\delta_{j}^{\prime}-\delta_{j}\right)\left(1-\delta_{j}^{\prime}\right)^{-1}$. Then $\delta_{j}^{\prime}=O\left(\vartheta_{j} a_{j}\right)$ by estimating term by term using $\nu_{j}=O\left(L^{-2 j} \vartheta_{j} a_{j}\right)$ and $w_{j}^{(1)}=O\left(L^{2 j}\right)$ by Theorem 2.1 and Lemma A.2. Also, $\Delta_{j}=O\left(\vartheta_{j} a_{j}^{2}\right)$ because $\delta_{j}^{\prime}-\delta_{j}=\left(\nu_{j+1}-\nu_{j}^{+}\right) w_{j+1}^{(1)}$ and there is a cancellation of all first order terms in $\nu_{j+1}-\nu_{j}^{+}$. 
By definition,

$$
\Pi_{j}=\prod_{k=0}^{j}\left(1-\delta_{k}^{\prime}\right) \prod_{i=0}^{j}\left(1+\Delta_{i}\right)=\exp \left[\sum_{k=0}^{j} \log \left(1-\delta_{k}^{\prime}\right)\right] \exp \left[\sum_{i=0}^{j} \log \left(1+\Delta_{i}\right)\right] .
$$

We use a telescoping sum and $w_{0}^{(1)}=0$ to see that

$$
\sum_{k=0}^{j} \log \left(1-\delta_{k}^{\prime}\right)=-\nu_{j+1} w_{j+1}^{(1)}+\sum_{k=0}^{j}\left[\log \left(1-\delta_{k}^{\prime}\right)+\delta_{k}^{\prime}\right]
$$

The sum on the right-hand side has terms $O\left(\vartheta_{k} a_{k}^{2}\right)$ so it is summable by (4.6), the infinite sum is a constant $c=O\left(a_{0}\right)$ and the sum over $k>j$ is $O\left(\vartheta_{j} a_{j}\right)$. Thus, with $\alpha=e^{c}$, we have

$$
\Pi_{j}=e^{-\nu_{j+1} w_{j+1}^{(1)}} \alpha e^{O\left(\vartheta_{j} a_{j}\right)},
$$

which has the desired form since $\nu_{j+1} w_{j+1}^{(1)}=O\left(\vartheta_{j} a_{j}\right)$.

We define $\lambda_{x, j}^{*}$ to be the sequence $\lambda_{x, j}$ when $\lambda_{x, 0}=1, \lambda_{y, 0}=0$ and $j_{x y}=\infty$. As in $[4,35]$, the sequence $\lambda_{x, j}$ with $\lambda_{x, 0}=\lambda_{y, 0}=1$ is equal to $\lambda_{x, j}^{*}$ until the coalescence scale.

Proposition 4.4. For $N \in \mathbb{N}$ and $j \leq N, \lambda_{\mathrm{x}, j}^{*}$ is continuous in $\left(m^{2}, a_{0}\right) \in[0, \delta)^{2}$ and

$$
\lambda_{\mathrm{x}, j}^{*}=1+O\left(\vartheta_{j} a_{j}\right)
$$

and similarly for $\lambda_{\mathrm{y}, j}^{*}$. In particular, $\lambda_{x, j_{x y}}=1+O\left(\vartheta_{j_{x y}} a_{j_{x y}}\right)$ for $x=\mathrm{x}, \mathrm{y}$.

Proof. We first use induction on $j$ to prove that

$$
\lambda_{x, j+1}^{*}=\Pi_{j}\left(1+\sum_{k=0}^{j} e_{k}\right), \quad\left|e_{k}\right| \leq 2 M \vartheta_{k} a_{k}^{2} \quad(j+1 \leq N) .
$$

If (4.26) holds for $j$ (it clearly holds for $j=0$ ), then, by (4.18),

$$
\lambda_{x, j+1}^{*}=\left(1-\delta_{j}\right) \Pi_{j-1}\left(1+\sum_{k=0}^{j} e_{k}\right)
$$

with $e_{j}=\Pi_{j}^{-1} R_{j+1}^{\lambda_{x}}$. This completes the induction, since by (4.19) and Lemma 4.3,

$$
\left|e_{j}\right| \leq 2\left|R_{j+1}^{\lambda_{x}}\right| \leq 2 M \vartheta_{j} a_{j}^{2}
$$

By $(2.25)$,

$$
\hat{\chi}_{N}=\frac{1}{m^{2}} \frac{\partial_{\sigma_{x}} D Z_{N}(0 ; \hat{\mathbb{1}})}{Z_{N}^{\varnothing}(0)} .
$$


As in the proof of Corollary 2.3, $\lim _{N \rightarrow \infty} Z_{N}^{\varnothing}(0)=1$. The $\sigma_{\mathrm{x}}$ term in $e^{-V_{N}(\varphi)}$ is simply $\lambda_{\mathrm{x}, N}^{*} \sigma_{\mathrm{x}}(\hat{1} \cdot \varphi)$, so its double derivative (with respect to $\sigma_{x}$ and with respect to $\varphi$ in the direction $\hat{\mathbb{1}}$ ) is $\lambda_{x, N}^{*}$. Since $\partial_{\sigma_{\mathrm{x}}} D Z_{N}=\partial_{\sigma_{\mathrm{x}}} D I_{N}+\partial_{\sigma_{\mathrm{x}}} D K_{N}$, we can use Theorem 2.2 to take the limit $N \rightarrow \infty$ and obtain

$$
\hat{\chi}=m^{-2} \lambda_{\mathrm{x}, \infty}^{*} \text {. }
$$

Thus, from (2.53) we conclude that $\lambda_{x, \infty}^{*}=1$. By (4.26), Lemma 4.3, and (4.6), this implies that

$$
\begin{aligned}
\lambda_{x, j+1}^{*}-1 & =\alpha\left(1+O\left(\vartheta_{j} a_{j}\right)\right)\left(1+\sum_{k=0}^{j} e_{k}\right)-\alpha\left(1+\sum_{k=0}^{\infty} e_{k}\right) \\
& =-\alpha \sum_{k=j+1}^{\infty} e_{k}+O\left(\vartheta_{j} a_{j}\right)=O\left(\vartheta_{j} a_{j}\right) .
\end{aligned}
$$

The remaining item is the continuity. The continuity can be concluded along the lines of the corresponding argument in the proof of [4, Proposition 4.3], and we omit the details.

\subsubsection{Flow of $q$}

The perturbative flow of $q$ is exactly as in [35, Proposition 3.2] (or [6, (3.35)] for $n=0$ ), namely, for $x=\mathrm{x}, \mathrm{y}$,

$$
q_{x, \mathrm{pt}}=q_{x}+\lambda_{\mathrm{x}} \lambda_{\mathrm{y}} C_{j+1 ; \times, \mathrm{y}}
$$

with $j$ the scale of the input $V$. Since $C_{j+1 ; a, b}=0$ when $j+1 \leq j_{\mathrm{xy}}$, for $j \leq j_{\mathrm{xy}}$ we have $q_{\mathrm{pt}}=0$ if $q=0$. Thus, while the perturbative flow of $\lambda$ stops at the coalescence scale, the flow of $q$ only starts at the coalescence scale. By Theorem 2.2, the nonperturbative flow also starts at the coalescence scale, and

$$
q_{x, j+1}=q_{x, j}+\lambda_{x, j_{x y}} \lambda_{y, j \times y} C_{j+1 ; \times, y}+R_{j+1}^{q_{x}} \quad\left(j+1 \geq j_{\times y}\right),
$$

with

$$
\left|R_{j+1}^{q_{x}}\right| \leq \mathbb{1}_{j+1 \geq j_{x y}}|\mathrm{x}-\mathrm{y}|^{-1} 4^{-\left(j-j_{x y}\right)} O\left(\vartheta_{j} a_{j}\right) .
$$

Proposition 4.5. Let $\lambda_{\mathrm{x}, 0}=\lambda_{\mathrm{y}, 0}=1$. For $\left(m^{2}, a_{0}\right) \in[0, \delta)^{2}$ and $x=\mathrm{x}, \mathrm{y}$, the limit

$$
q_{x, \infty}\left(m^{2}, a_{0}\right)=\lim _{N \rightarrow \infty} q_{x, N}\left(m^{2}, a_{0}\right)
$$

exists, is continuous, and, as $|\mathrm{x}-\mathrm{y}| \rightarrow \infty$,

$$
q_{x, \infty}\left(0, a_{0}\right)=\left(-\Delta_{\mathbb{Z}^{3}}^{-1}\right)_{x, y}\left(1+O\left(\frac{1}{\log |\mathrm{x}-\mathrm{y}|}\right)\right) .
$$

Proof. For $N \in \mathbb{N}$ and $\left(m^{2}, g_{0}\right) \in\left[L^{-2(N-1)}, \delta\right) \times(0, \delta)$, the solution of the recursion (4.33) is

$$
q_{x, N}=\lambda_{x, j_{x y}} \lambda_{y, j_{x y}} w_{N ; \times, y}+\sum_{i=j_{x y}}^{N-1} R_{i+1}^{q_{x}} .
$$


The limit $N \rightarrow \infty$ exists, and by Proposition 4.4, (4.34), and Proposition 4.1,

$$
\begin{aligned}
q_{x, \infty}\left(m^{2}, a_{0}\right) & =\lambda_{x, j_{x y}} \lambda_{y, j_{x y}} C_{x, y}\left(m^{2}\right)+\sum_{i=j_{x y}}^{\infty} R_{i+1}^{q_{x}} \\
& =\left(1+O\left(\vartheta_{j_{x y}} a_{j_{x y}}\right)\right) C_{x, y}\left(m^{2}\right)+|x-y|^{-1} O\left(\vartheta_{j_{x y}} a_{j_{x y}}\right) \\
& =\left(1+O\left(j_{x y}^{-1}\right)\right) C_{x, y}\left(m^{2}\right)+|x-y|^{-1} O\left(j_{x y}^{-1}\right) .
\end{aligned}
$$

The continuity is a consequence of the continuity of $C_{\mathrm{x}, \mathrm{y}}, \lambda_{x, \mathrm{xy}}$ and $R^{q_{x}}$, and the estimate (4.36) follows from $j_{\mathrm{xy}} \asymp_{L} \log |\mathrm{x}-\mathrm{y}|$ and the fact that $C_{\mathrm{x}, \mathrm{y}}(0)=\left(-\Delta_{\mathbb{Z}^{3}}\right)_{\mathrm{x}, \mathrm{y}}^{-1}$.

\section{Proof of Theorems 1.1-1.2}

\subsection{Proof of Theorem 1.1}

Fix $\left(m^{2}, a\right) \in(0, \delta)$. Let $\left(a_{0}, g_{0}, \nu_{0}, z_{0}\right)$ be given by the continuous functions $\left(a_{0}^{*}, g_{0}^{*}, \nu_{0}^{*}, z_{0}^{*}\right)$ of Proposition 2.4; we record this with notational stars in the following. By (2.24), and since $W_{N}$ has no $\sigma_{\mathrm{x}} \sigma_{\mathrm{y}}$ term by [15, Proposition 4.10],

$$
\begin{aligned}
G_{N ; \times, y}\left(a, g^{*}, \nu^{*} ; n\right) & =\left(1+z_{0}^{*}\right) \partial_{\sigma_{x} \sigma_{\mathrm{y}}}^{2} \log Z_{N}^{*}(0) \\
& =\left(1+z_{0}^{*}\right) \partial_{\sigma_{\times} \sigma_{\mathrm{y}}}^{2} \log \left(e^{\frac{1}{2}\left(q_{\mathrm{x}, N}^{*}+q_{\mathrm{y}, N}^{*}\right) \sigma_{\times} \sigma_{\mathrm{y}}}\left(1+W_{N}^{*}(0)\right)+K_{N}^{*}(0)\right) \\
& =\left(1+z_{0}^{*}\right)\left(\frac{1}{2}\left(q_{\mathrm{x}, N}^{*}+q_{\mathrm{y}, N}^{*}\right)+\partial_{\sigma_{\times} \sigma_{\mathrm{y}}}^{2} \log \left(1+K_{N}^{*}(0)\right)\right) .
\end{aligned}
$$

By Proposition 4.5 and Theorem 2.2, the $N \rightarrow \infty$ limit of the right-hand side exists and is

$$
G_{\mathrm{x}, \mathrm{y}}\left(a, g^{*}, \nu^{*} ; n\right)=\left(1+z_{0}^{*}\right) \frac{1}{2}\left(q_{\mathrm{x}, \infty}^{*}+q_{\mathrm{y}, \infty}^{*}\right) .
$$

Since $z_{0}^{*}$ and $q_{x, \infty}^{*}$ are continuous as $m^{2} \downarrow 0$, from (4.36) we obtain

$$
\begin{aligned}
G_{\mathrm{x}, \mathrm{y}}\left(a, g^{*}(0, a), \nu^{*}(0, a) ; n\right) & =\left(1+z_{0}^{*}(0, a)\right) \frac{1}{2}\left(q_{\mathrm{x}, \infty}\left(0, a_{0}^{*}(0, a)\right)+q_{\mathrm{y}, \infty}\left(0, a_{0}^{*}(0, a)\right)\right) \\
& =\left(1+z_{0}^{*}(0, a)\right)\left(-\Delta_{\mathbb{Z}^{3}}^{-1}\right)_{\mathrm{x}, \mathrm{y}}\left(1+O\left(\frac{1}{\log |\mathrm{x}-\mathrm{y}|}\right)\right) .
\end{aligned}
$$

This proves $(1.17)$ with $A_{a, n}=\left(1+z^{*}(0, a)\right)(4 \pi)^{-1}$, since $\left(-\Delta_{\mathbb{Z}^{3}}\right)_{\mathrm{x}, \mathrm{y}}^{-1}=\frac{1}{4 \pi}|\mathrm{x}-\mathrm{y}|^{-1}\left(1+O\left(|\mathrm{x}-\mathrm{y}|^{-2}\right)\right)$ (see, e.g., [28], the constant $(4 \pi)^{-1}$ is for our definition of the Laplacian). The tricritical point is $\left(g^{*}(0, a), \nu^{*}(0, a)\right)$, and the continuous curve in Theorem 1.1 is the curve $\left(g^{*}\left(m^{2}, a\right), \nu^{*}\left(m^{2}, a\right)\right)$ parametrised by $m^{2}$.

\subsection{Proof of Theorem $\mathbf{1 . 2}$}

Throughout this section we take $m^{2}=0$, and we write $\simeq$ for equality up to an additive term that is $O\left(\mu_{3,0}^{2}\right)$. 
It suffices to prove that

$$
\begin{aligned}
& \mu_{2,0} \simeq-\frac{3}{2}(n+4) C_{0,0} \mu_{3,0}, \\
& \mu_{1,0} \simeq \frac{3}{4}(n+4)(n+2) C_{0,0}^{2} \mu_{3,0} .
\end{aligned}
$$

The proof is similar to the analysis in [5, Section 8.5]. Our starting point is the equations

$$
\begin{aligned}
& \mu_{2, j}=-\sum_{l=j}^{\infty} L^{-(l-j)} \pi_{j, l}^{-1}\left(\bar{\beta}_{2, l}^{3} \mu_{3, l}+O\left(\vartheta_{l} \mu_{3, l}^{2}\right)\right), \\
& \mu_{1,0}=-\sum_{l=0}^{\infty} L^{-2 l}\left(\bar{\beta}_{1, l}^{2} \mu_{2}+\bar{\beta}_{1, l}^{3} \mu_{3}+O\left(\vartheta_{l} \mu_{3, l}^{2}\right)\right),
\end{aligned}
$$

which are consequences of (4.11) and (4.14). Here,

$$
\pi_{j, l}^{-1}=\left(\frac{\mu_{3, j}}{\mu_{3, l+1}}\right)^{p_{2}}\left(c_{j}^{-1}+O\left(\vartheta_{l} \mu_{3, l}\right)\right) \quad \text { with } \quad c_{j}^{-1}=1+O\left(\vartheta_{j} \mu_{3, j}\right),
$$

and

$$
L^{-2 l} \bar{\beta}_{1, l}^{2}=b_{1}^{2} C_{l+1}, \quad L^{-2 l} \bar{\beta}_{1, l}^{3}=b_{1}^{3} C_{l+1}^{2}, \quad L^{-l} \bar{\beta}_{2, l}^{3}=b_{2}^{3} C_{l+1}
$$

with $C_{l+1}=C_{l+1 ; 0,0}$ and

$$
b_{1}^{2}=(n+2), \quad b_{1}^{3}=\frac{3}{4}(n+4)(n+2), \quad b_{2}^{3}=\frac{3}{2}(n+4) .
$$

Note that $b_{1}^{2} b_{2}^{3}=2 b_{1}^{3}$.

Proof of Theorem 1.2. By (5.6), (4.13), and Lemma 4.2,

$$
\begin{aligned}
\mu_{2,0} & \simeq-b_{2}^{3} \sum_{j=0}^{\infty} \pi_{0, j}^{-1} C_{j+1} \mu_{3, j} \\
& =-b_{2}^{3}\left(1+O\left(\mu_{3,0}\right)\right) \mu_{3,0}^{p_{2}} \sum_{j=0}^{\infty} C_{j+1} \mu_{3, j}^{1-p_{2}} \simeq-b_{2}^{3} \mu_{3,0} C_{0,0},
\end{aligned}
$$

which proves (5.4). Similarly, by (5.6)-(5.7) (we use $b_{1}^{2} b_{2}^{3}=2 b_{1}^{3}$ to obtain the third line),

$$
\begin{aligned}
\mu_{1,0} & \simeq-b_{1}^{2} \sum_{j=0}^{\infty} C_{j+1} \mu_{2, j}-b_{1}^{3} \sum_{j=0}^{\infty} C_{j+1}^{2} \mu_{3, j} \\
& \simeq b_{1}^{2} b_{2}^{3} \sum_{j=0}^{\infty} C_{j+1} \sum_{l=j}^{\infty} \pi_{l, j}^{-1} C_{l+1} \mu_{3, l}-b_{1}^{3} \sum_{j=0}^{\infty} C_{j+1}^{2} \mu_{3, j} \\
& \simeq b_{1}^{3}\left(\sum_{j=0}^{\infty} 2 C_{j+1} \sum_{l=j+1}^{\infty} \pi_{l, j}^{-1} C_{l+1} \mu_{3, l}+\sum_{j=0}^{\infty} C_{j+1}^{2} \mu_{3, j}\right) \\
& =b_{1}^{3} \sum_{l=0}^{\infty}\left(2 C_{l+1} \sum_{j=0}^{l-1} \pi_{j, l}^{-1} C_{j+1}+C_{l+1}^{2}\right) \mu_{3, l} .
\end{aligned}
$$


By Lemma 4.2,

$$
\sum_{l=0}^{\infty} C_{l+1}^{2} \mu_{3, l} \simeq \mu_{3,0} \sum_{l=0}^{\infty} C_{l+1}^{2}
$$

Also,

$$
\begin{aligned}
2 \sum_{l=0}^{\infty} C_{l+1} \sum_{j=0}^{l-1} \pi_{j, l}^{-1} C_{j+1} \mu_{3, l} & =\left(2+O\left(\mu_{3,0}\right)\right) \sum_{l=0}^{\infty} C_{l+1} \mu_{3, j}^{p_{2}} \sum_{j=0}^{l-1} C_{j+1} \mu_{3, l}^{1-p_{2}} \\
& =\left(2+O\left(\mu_{3,0}\right)\right) \sum_{l=0}^{\infty} C_{l+1} \mu_{3, j}^{p_{2}}\left(\sum_{j=0}^{l-1} C_{j+1} \mu_{3,0}^{1-p_{2}}+O\left(\mu_{3,0}^{2-p_{2}}\right)\right) \\
& \simeq 2 \mu_{3,0} \sum_{l=0}^{\infty} C_{l+1} \sum_{j=0}^{l-1} C_{j+1}=\mu_{3,0}\left(C_{0,0}^{2}-\sum_{l=0}^{\infty} C_{l+1}^{2}\right)
\end{aligned}
$$

Therefore, there is a cancellation and

$$
\mu_{1,0} \simeq b_{1}^{3} C_{0,0}^{2} \mu_{3,0},
$$

which is (5.5). This completes the proof.

\section{A Covariance decomposition}

Finite-range covariance decompositions of the type we use were developed in [12], and a different perspective was given in [2] which we follow here. The following lemma is a restatement of $[6$, Proposition 6.1]. The $C_{j}$ are positive-definite on $\mathbb{Z}^{d}$, as discussed below [31, (1.7)]. This does not immediately imply that they are positive-definite on the torus, but they are at least positive semi-definite and this is sufficient for our needs.

Let $\nabla_{x_{k}}$ denote the finite-difference operator $\nabla_{x_{k}} f(x, y)=f\left(x+e_{k}, y\right)-f(x, y)$, where $e_{k}$ is one of the $2 d$ (positive or negative) unit vectors on $\mathbb{Z}^{d}$. We write $\nabla_{x}^{\alpha}=\nabla_{x_{1}}^{\alpha_{1}} \cdots \nabla_{x_{d}}^{\alpha_{d}}$ for a multi-index $\alpha=\left(\alpha_{1}, \ldots, \alpha_{d}\right) \in \mathbb{N}_{0}^{d}$.

Proposition A.1. Let $d>2, L \geq 2, j \geq 1, m^{2} \in[0,1]$. There exist positive-definite covariances $C_{j}$ on $\mathbb{Z}^{d}$ such that $\left(-\Delta+m^{2}\right)^{-1}=\sum_{j=1}^{\infty} C_{j}$, and the following hold.

(i) For multi-indices $\alpha, \beta$ with $\ell^{1}$ norms $|\alpha|_{1},|\beta|_{1}$ at most some fixed value $p$, and for any $q>0$,

$$
\left|\nabla_{x}^{\alpha} \nabla_{y}^{\beta} C_{j ; x, y}\right| \leq c\left(1+m^{2} L^{2(j-1)}\right)^{-q} L^{-(j-1)\left(d-2+\left(|\alpha|_{1}+|\beta|_{1}\right)\right)},
$$

where $c=c(p, q)$ is independent of $m^{2}, j, L$. If $m^{2} L^{2(N-1)} \geq \varepsilon$ for some $\varepsilon>0$, then the same bound holds for $C_{N, N}$ with $c$ depending on $\varepsilon$ but independent of $N$.

(ii) The $C_{j}$ have the finite-range property $C_{j ; x, y}=0$ if $|x-y| \geq \frac{1}{2} L^{j}$.

(iii) Let $m^{2}=0$. There exists a smooth function $c_{0}: \mathbb{R}^{d} \rightarrow \mathbb{R}$ with compact support such that, as $j \rightarrow \infty$,

$$
C_{j ; x, y}=L^{-(d-2) j} c_{0}\left(L^{-j}(x-y)\right)+O\left(L^{-(d-1)(j-1)}\right) \quad \text { for } m^{2}=0 .
$$


The next lemma concerns the quantities defined in (3.62). Recall that the mass scale $j_{m}$ is the largest integer $j$ such that $m L^{j} \leq 1$, with $j_{0}=\infty$.

Lemma A.2. For $d=3, j \geq 1$, and $m^{2} \in[0,1]$,

$$
\begin{aligned}
w_{j}^{(k)} & =O_{L}\left(L^{(3-k)\left(j \wedge j_{m}\right)}\right) & & (k<3), \\
w_{j}^{(k, * *)} & =O_{L}\left(L^{(5-k)\left(j \wedge j_{m}\right)}\right) & & (k<5), \\
\left(\Delta w_{j}\right)^{(k, * *)} & =O_{L}\left(L^{(3-k)\left(j \wedge j_{m}\right)}\right) & & (k<3) .
\end{aligned}
$$

Proof. The factor involving the mass in (A.1) obeys $\left(1+m^{2} L^{2(j-1)}\right)^{-q} \leq O_{L}\left(L^{-q\left(j-j_{m}\right)+}\right)$, so $C_{j ; 0, x}=$ $O\left(L^{-j} L^{-q\left(j-j_{m}\right)+}\right)$. For $k=1$, with the finite-range property we obtain

$$
w_{j}^{(1)}=\sum_{1 \leq i \leq j} \sum_{x} C_{i ; 0, x} \leq \sum_{1 \leq i \leq j} L^{3 i} L^{-i} O_{L}\left(L^{-q\left(i-j_{m}\right)+}\right) \leq O_{L}\left(L^{2\left(j \wedge j_{m}\right)}\right),
$$

and, for $k=2$,

$$
\begin{aligned}
w_{j}^{(2)} & \leq 2 \sum_{1 \leq i \leq l \leq j} \sum_{x} C_{i ; 0, x} C_{l ; 0, x} \leq \sum_{1 \leq i \leq l \leq j} L^{3 i} L^{-i} L^{-q\left(i-j_{m}\right)_{+}} L^{-l} O_{L}\left(L^{-q\left(l-j_{m}\right)_{+}}\right) \\
& \leq \sum_{1 \leq i \leq j} L^{2 i} L^{-q\left(i-j_{m}\right)_{+}} L^{-i} O_{L}\left(L^{-q\left(i-j_{m}\right)_{+}}\right) \leq O_{L}\left(L^{\left(j \wedge j_{m}\right)}\right) .
\end{aligned}
$$

The proof for $\left(\Delta w_{j}\right)^{(k, * *)}$ is the same because $\sum_{x} C_{i ; 0, x}$ and $\sum_{x} x_{1}^{2} \Delta C_{i ; 0, x}$ obey the same bounds $L^{3 i} L^{-i}=L^{2 i}$ and $L^{3 i} L^{2 i} L^{-3 i}=L^{2 i}$ (with the same enhanced decay beyond the mass scale).

The proof for $w_{j}^{(k, * *)}$ is almost the same for $k=1,2$ as it is for $w_{j}^{(n)}$ : we simply replace the $L^{3 i} L^{-i}$ bound on $\sum_{x} C_{i ; 0, x}$ by a bound with an additional factor $L^{2 i}$. For $k=3,4$ it is similar.

With further effort, the constant $\lambda_{3}$ in Lemma A.3 could be computed as an explicit universal constant times $\log L$, as in [6, Lemma 6.3(a)].

Lemma A.3. Let $d=3$ and $m^{2}=0$. There is an L-dependent constant $\lambda_{3}$ such that

$$
\lim _{j \rightarrow \infty}\left(w_{j+1}^{(3)}-w_{j}^{(3)}\right)=\lambda_{3} .
$$

Proof. The proof is similar to the proof of [6, Lemma 6.3(a)] for $w^{(2)}$ when $d=4$, so we only give a sketch. Constants in error estimates may depend on $L$ here, and we abbreviate subscripts $0, x$ by $x$ alone. Sums are over $x \in \mathbb{Z}^{3}$ and integrals are over $\mathbb{R}^{3}$.

Since $w_{j+1}=w_{j}+C_{j+1}$, we have

$$
\begin{aligned}
w_{j+1}^{(3)}-w_{j}^{(3)} & =\sum_{x}\left(C_{j+1 ; x}^{3}+3 C_{j+1 ; x}^{2} w_{j ; x}+3 C_{j+1 ; x} w_{j ; x}^{2}\right) \\
& =\sum_{x} C_{j+1 ; x}^{3}+3 \sum_{k=1}^{j} \sum_{x} C_{j+1 ; x}^{2} C_{k ; x}+3 \sum_{k, l=1}^{j} \sum_{x} C_{j+1 ; x} C_{k ; x} C_{l ; x} .
\end{aligned}
$$


We use (A.2) to write $C_{i ; x}=c_{i}(x)+e_{i}(x)$ with $c_{i}(x)=L^{-i} c_{0}\left(L^{-i} x\right)$ and $e_{i}(x)=O\left(L^{-2 i}\right)$. By Riemann summation,

$$
\begin{aligned}
\sum_{x} c_{k}(x) c_{k+s}(x) c_{k+t}(x) & =L^{-3 k} \sum_{x} c_{0}\left(L^{-k} x\right) c_{s}\left(L^{-k} x\right) c_{t}\left(L^{-k} x\right) \\
& =\int c_{0}(x) c_{s}(x) c_{t}(x) d x+O\left(L^{-k-s-t}\right)
\end{aligned}
$$

where the error term is a bound on $L^{-k}\left\|c_{0} c_{s} c_{t}\right\|_{\infty}$. This permits the sums over $x$ in (A.9) to be approximated by integrals, and the sums of those integrals over scales produce the constant $\lambda_{3}$. For example, the first term on the right-hand side of (A.9) is

$$
\sum_{x} C_{j+1 ; x}^{3}=\sum_{x} c_{j+1}(x)^{3}+\sum_{x} e_{j+1}(x)\left(3 c_{j+1}^{2}(x)+3 c_{j+1}(x) e_{j+1}(x)+e_{j+1}^{2}(x)\right) .
$$

The first term is equal to $\int c_{0}^{3}$ plus an error of order $L^{-j}$, and the remaining sum is smaller by $L^{-j}$ because there is at least one $e_{j+1}$ factor. Similarly, the middle term in (A.9) is handled by

$$
\sum_{k=1}^{j} \sum_{x} C_{j+1 ; x}^{2} C_{k ; x}=\sum_{l=1}^{j} \int c_{l}^{2} c_{0}+O\left(L^{-j}\right)
$$

together with the fact that the series $\sum_{l=1}^{\infty} \int c_{l}^{2} c_{0}$ converges since its terms are $O\left(L^{-2 l}\right)$. The last term in (A.9) can be handled similarly.

\section{B Existence of a global RG flow}

We discuss the minor changes to the analysis of $[4,7,15,16]$ that lead to a proof of Theorems 2.1-2.2.

\section{B.1 Parameters}

Several parameters require natural adjustments to take into account the change from $|\varphi|^{4}$ and $d=4$ to $|\varphi|^{6}$ and $d=3$.

\section{B.1.1 Norm parameters}

The space $\mathcal{K}$ is as in [35, Definition 4.5] with $h=\hat{1}$, and $\mathcal{C K}$ is its restriction to connected polymers. We modify the definition of the $\mathcal{W}$-norm on $\mathcal{C K}$, with the following revised parameters compared to $\left[16\right.$, Section 1.7]. We fix a sequence $\tilde{a}_{j}$ such that

$$
\frac{1}{2} \tilde{a}_{j+1} \leq \tilde{a}_{j} \leq 2 \tilde{a}_{j+1}
$$

Specifically, given $\tilde{m}^{2} \geq 0$, we use the sequence

$$
\tilde{a}_{j}\left(\tilde{m}^{2}, \tilde{a}_{0}\right)=\bar{a}_{j}\left(0, \tilde{a}_{0}\right) \mathbb{1}_{j \leq j \tilde{m}}+\bar{a}_{j_{\tilde{m}}}\left(0, \tilde{a}_{0}\right) \mathbb{1}_{j>j_{\tilde{m}}}
$$


where $j_{m}$ is the mass scale, and $\bar{a}$ is the sequence $\mu_{3}$ of (4.1) with remainder $e_{3}=0$ and initial condition $\bar{a}_{0}=\tilde{a}_{0}$. The norm depends on $\tilde{m}^{2}$, which is a replacement for $m^{2}$ to avoid dependence of the norm on $\mathrm{m}^{2}$; see the discussion above [16, Theorem 1.13].

Given a (large) $L$-dependent constant $\ell_{0}$ and a (small) $L$-independent constant $k_{0}$, we set

$$
\begin{gathered}
\mathfrak{h}_{j}= \begin{cases}\ell_{0} L^{-j / 2} & (\mathfrak{h}=\ell) \\
k_{0} \tilde{a}_{j}^{-1 / 6} L^{-d j / 6} & (\mathfrak{h}=h),\end{cases} \\
\mathfrak{h}_{\sigma, j}=\ell_{j \wedge j_{\times y}}^{-1} 2^{\left(j-j_{\times y}\right)_{+}} \times \begin{cases}\tilde{a}_{j} & (\mathfrak{h}=\ell) \\
\tilde{a}_{j}^{1 / 6} & (\mathfrak{h}=h) .\end{cases}
\end{gathered}
$$

With $\tilde{\vartheta}_{j}=2^{-\left(j-j_{\tilde{m}}\right)_{+}}$, we define

$$
\bar{\epsilon}_{j}= \begin{cases}\tilde{\vartheta}_{j} \tilde{a}_{j} & (\mathfrak{h}=\ell) \\ \tilde{\vartheta}_{j} \tilde{a}_{j}^{1 / 6} & (\mathfrak{h}=h)\end{cases}
$$

and set

$$
\omega_{j}=\frac{\bar{\epsilon}_{j}(\ell)}{\bar{\epsilon}_{j}(h)}=\tilde{a}_{j}^{5 / 6}
$$

With the above parameters, exactly as in $[16$, Section 1.7$]$ we define a norm on $\mathcal{C} \mathcal{K}_{j}$ by

$$
\|K\|_{\mathcal{W}_{j}}=\max \left\{\|K\|_{\mathcal{F}_{j}(G)}, \omega_{j}^{3}\|K\|_{\mathcal{F}_{j}(\tilde{G})}\right\}
$$

In $[16,(1.44)]$ for $d=4$, (B.6) was instead $\omega_{j}=\tilde{g}_{j}^{3 / 4}$ so $\omega_{j}^{3}=\tilde{g}_{j}^{9 / 4}=O\left(\ell_{j} / h_{j}\right)^{9}$ and $\omega_{j}^{3}\left(\tilde{g}_{j}^{1 / 4}\right)^{3}=\tilde{g}_{j}^{3}$. This determined that we needed $p_{\mathcal{N}} \geq 9+1=10$ derivatives of the field (see [16, Lemma 2.4]). Now, instead, we have $\omega_{j}^{3}$ of order $\left(\ell_{j} / h_{j}\right)^{15}$, and we can choose any $p_{\mathcal{N}} \geq 16$.

Also, a scale-dependent norm on $\mathcal{U} \simeq \mathbb{C}^{10}$ is defined by

$$
\|U\|_{\mathcal{U}}=\max \left\{|a|,|z|,|y|,|g| L^{j},|\nu| L^{2 j},|u| L^{3 j}, \ell_{j} \ell_{\sigma, j}\left|\lambda_{\times}\right|, \ell_{j} \ell_{\sigma, j}\left|\lambda_{\mathrm{y}}\right|, \ell_{\sigma, j}^{2}\left|q_{\mathbf{x}}\right|, \ell_{\sigma, j}^{2}\left|q_{\mathrm{y}}\right|\right\},
$$

with $\ell_{j}$ and $\ell_{\sigma, j}$ as in (B.3)-(B.4). The RG domain for $V$ now becomes, with $C_{\mathcal{D}}$ a (large) universal constant,

$$
\begin{gathered}
\mathcal{D}_{j}=\left\{\left(a, g, \nu, z, \lambda_{a}, \lambda_{b}\right) \in \mathbb{C}^{6}: \operatorname{Re} a>C_{\mathcal{D}}^{-1} \tilde{a},|\operatorname{Im} a|<\frac{1}{10} C_{\mathcal{D}} \tilde{a},\right. \\
\left.L^{j}|g|, L^{2 j}|\nu|,|z| \leq C_{\mathcal{D}} \tilde{a},\left|\lambda_{a}\right|,\left|\lambda_{b}\right| \leq C_{\mathcal{D}}\right\} .
\end{gathered}
$$

The stability domains $[15,(1.85)-(1.86)]$ are replaced now, given $\alpha, \alpha^{\prime}, \alpha_{L}^{\prime \prime}>0$, by

$$
\begin{aligned}
& \overline{\mathcal{D}}_{j}(\ell)=\left\{V:|\operatorname{Im} a|<\frac{1}{5} \operatorname{Re} a, \epsilon_{V, j}\left(\ell_{j}\right) \leq \alpha_{L}^{\prime \prime} \tilde{a}\right\}, \\
& \overline{\mathcal{D}}_{j}(h)=\left\{V:|\operatorname{Im} \tilde{a}|<\frac{1}{5} \operatorname{Re} \tilde{a}, \alpha \leq \epsilon_{a \tau^{3}, j}\left(h_{j}\right), \epsilon_{V, j}\left(h_{j}\right) \leq \alpha^{\prime}\right\} .
\end{aligned}
$$

\section{B.1.2 Small parameters $\epsilon_{V}$ and $\bar{\epsilon}$}

The small parameter $\epsilon_{V}$, defined in [15, (1.80)] as a sum of $T_{0}$-seminorms of monomials, requires modification in our present setting. The parameters $\mathfrak{h}$ and $\mathfrak{h}_{\sigma}$ have been chosen precisely to make this modification insignificant. We illustrate this here for the monomials $a \tau^{3}, g \tau^{2}$, and $\nu \tau$ :

$$
L^{d j}\left(\left\|a \tau^{3}\right\|_{T_{0}(\mathfrak{h})}+\left\|g \tau^{2}\right\|_{T_{0}(\mathfrak{h})}+\|\nu \tau\|_{T_{0}(\mathfrak{h})}\right) \asymp \begin{cases}|a| \ell_{0}^{6}+L^{j}|g| \ell_{0}^{4}+L^{2 j}|\nu| \ell_{0}^{2} & (\mathfrak{h}=\ell) \\ k_{0}^{6}+L^{j}|g| a^{-4 / 6} k_{0}^{4}+L^{2 j}|\nu| a^{-2 / 6} k_{0}^{2} & (\mathfrak{h}=h) .\end{cases}
$$


For $V \in \mathcal{D}_{j}$, the right-hand side is bounded by an $L$-dependent multiple of $\tilde{a}$ when $\mathfrak{h}=\ell$, and by an $L$-independent multiple of $k_{0}$ when $\mathfrak{h}=h$.

Our choice of the small parameter $\bar{\epsilon}$ in (B.5) is made to dominate the norm of $\delta V$ as in $[15$, Lemma 3.4]. By following the proof of [15, Lemma 3.4], for $V \in \overline{\mathcal{D}}$ we again get, as required,

$$
\begin{aligned}
\|\delta V(b)\|_{T_{0}(\mathfrak{h} \sqcup \ell)} & \leq\|\theta V(b)-V(b)\|_{T_{0}(\mathfrak{h} \sqcup \ell)}+\left\|V(b)-V_{\mathrm{pt}}(b)\right\|_{T_{0}(\mathfrak{h} \sqcup \ell)} \\
& \leq \frac{\hat{\ell}}{\mathfrak{h}} O_{L}\left(\epsilon_{V}\right) \leq O_{L}(\bar{\epsilon}) .
\end{aligned}
$$

\section{B.1.3 Localisation parameters}

By definition, the operator $\operatorname{Loc}_{X}$ acts term by term in the direct sum decomposition (2.26), with an action that depends on the number of $\sigma$ factors (as well as on the scale when there is just one $\sigma)$. As discussed in detail in [14], the definitions require: (i) specification of the field dimensions, (ii) choice of a maximal monomial dimension $d_{+}(\alpha)$ for each $\alpha \in\{\varnothing, \mathrm{x}, \mathrm{y}, \mathrm{xy}\}$, and (iii) choice of covariant field polynomials $\hat{P}$. Item (iii) is done exactly as in $[14,(1.19)]$ and plays a minor role. The field dimension is always $\frac{d-2}{2}=\frac{1}{2}$ in this paper. For (ii), we make the following choices. For $\alpha=\varnothing$, we set $d_{+}(\varnothing)=d=3$. For $\alpha=\mathrm{xy}$, we set $d_{+}(\mathrm{xy})=0$. For $\alpha=\mathrm{x}$ and $\alpha=\mathrm{y}$, we make the scale dependent choice $d_{+}^{(j)}(\mathrm{x})=d_{+}^{(j)}(\mathrm{y})=\frac{1}{2} \mathbb{1}_{j<j_{x y}}$, where $j_{\mathrm{xy}}$ is the coalescence scale. This is as in $[4,35]$, after taking into account that the field dimension here is $\frac{1}{2}$ rather than 1 .

\section{B.2 Stability}

The delicate stability estimate is $\left[15\right.$, Proposition 5.1(ii)], which shows how the $|\varphi|^{4}$ term in $e^{-V}$ provides integrability for $d=4$. The next proposition adapts its essential part to our present setting, in which the $|\varphi|^{6}$ term stabilises the integral for $d=3$. Only minor modifications to the proof of $\left[15\right.$, Proposition 5.1(ii)] are needed. In addition to the $T_{\varphi}$-seminorm, the statement involves the same $\Phi$ and $\tilde{\Phi}$ norms used in [15].

Proposition B.1. Let $q \geq 0, B \in \mathcal{B}_{j}, V \in \mathcal{U}$. Suppose that $|\operatorname{Im} a| \leq \frac{1}{2} \operatorname{Re} a$ and $\epsilon_{V} \leq C \epsilon_{a \tau^{3}}$. Then

$$
\left\|e^{-V(B)}\right\|_{T_{\varphi}} \leq e^{O\left(1+q^{3}\right) \epsilon_{V}} e^{\left.-q \epsilon_{a \tau^{3}}\|\varphi\|_{\Phi(B}^{2}\right)^{2}} e^{O(q) \epsilon_{V}\|\varphi\|_{\tilde{\Phi}(B \square)}^{2}} .
$$

Proof. For notational simplicity, we present the proof for the case $n \geq 1$; a minor adaptation applies for $n=0$. Constants in this proof can depend on $n$.

We isolate the $|\varphi|^{6}$ term in $V$ as $V=\frac{1}{8} a|\varphi|^{6}+Q$. By the product property of the $T_{\varphi}$-seminorm,

$$
\left\|e^{-V(b)}\right\|_{T_{\varphi}} \leq e^{\|Q(b)\|_{T_{\varphi}}} \prod_{x \in B}\left\|e^{-\frac{1}{8} a \varphi_{x}^{6}}\right\|_{T_{\varphi}}
$$

Let $\alpha=\frac{1}{8} \operatorname{Re} a$, so $\frac{1}{8}|a| \leq \frac{3}{2} \alpha$ by hypothesis. Let $t_{x}=\left|\varphi_{x}\right| / \mathfrak{h}, H=(\mathfrak{h}, \ldots, \mathfrak{h})$, and $P(t)=(t+\sqrt{n})^{2}$. As in the proof of [13, Proposition 3.9],

$$
\left\|\left|\varphi_{x}\right|^{2}\right\|_{T_{\varphi}}=\left|\varphi_{x}+H\right|^{2} \leq \mathfrak{h}^{2} P\left(t_{x}\right)
$$

and

$$
\left\|e^{-\frac{1}{8} a \varphi_{x}^{6}}\right\|_{T_{\varphi}} \leq e^{-2 \alpha\left|\varphi_{x}\right|^{6}} e^{\frac{3}{2} \alpha \mathfrak{h}^{6} P\left(t_{x}\right)^{3}}=e^{\alpha \mathfrak{h}^{6}\left[-2 t_{x}^{6}+\frac{3}{2} P\left(t_{x}\right)^{3}\right]} .
$$


Given any $q_{1} \geq 0$, we can estimate the exponent on the right-hand side to obtain

$$
\left\|e^{-\frac{1}{8} a \varphi_{x}^{6}}\right\|_{T_{\varphi}} \leq e^{\alpha \mathfrak{h}^{6}\left[q_{2}-q_{1}\left(t_{x}^{2}+t_{x}^{4}\right)\right]}
$$

for some $q_{2}=O\left(1+q_{1}^{3}\right)$. We define $\|\varphi\|_{L^{p}}^{p}=L^{-d j} \sum_{x \in B}\left(\left|\varphi_{x}\right| / \mathfrak{h}\right)^{p}$. Then, since $\epsilon_{a \tau^{3}}=\frac{1}{8}|a| \mathfrak{h}^{6} L^{d j} \leq$ $\frac{3}{2} \alpha \mathfrak{h}^{6} L^{d j}$

$$
\prod_{x \in B}\left\|e^{-\frac{1}{8} a \varphi_{x}^{6}}\right\|_{T_{\varphi}} \leq e^{\left(O\left(1+q_{1}^{3}\right)-\frac{2}{3} q_{1}\left(\|\varphi\|_{L^{2}}^{2}+\|\varphi\|_{L^{4}}^{4}\right)\right) \epsilon_{a \tau^{3}}} .
$$

Since for some $c>0$ we have

$$
\|Q(b)\|_{T_{\varphi}} \leq c \epsilon_{V}\left(\|\varphi\|_{L^{4}}^{4}+1\right),
$$

and since $\epsilon_{V} \leq C \epsilon_{a \tau^{3}}$ by hypothesis, it follows that

$$
\log \left\|e^{-V(b)}\right\|_{T_{\varphi}} \leq\left[O\left(1+q_{1}^{3}\right)-\frac{2}{3} q_{1}\|\varphi\|_{L^{2}}^{2}-\frac{2}{3} q_{1}\|\varphi\|_{L^{4}}^{4}+c C\left(\|\varphi\|_{L^{4}}^{4}+1\right)\right] \epsilon_{a \tau^{3}} .
$$

Now we choose $q_{1}=\frac{3}{2}(q+c C)$ to obtain

$$
\log \left\|e^{-V(b)}\right\|_{T_{\varphi}} \leq O\left(1+q^{3}\right) \epsilon_{V}-q\|\varphi\|_{L^{2}}^{2} \epsilon_{a \tau^{3}} .
$$

Finally, as the proof of [15, Proposition 5.1(ii)] we use

$$
\|\varphi\|_{L^{2}}^{2} \geq \frac{1}{2 c_{2}^{2}}\|\varphi\|_{\Phi\left(B^{\square}\right)}^{2}-\|\varphi\|_{\tilde{\Phi}\left(B^{\square}\right)}^{2},
$$

and redefine $q$ to complete the proof.

\section{B.3 Proof of Theorems 2.1-2.2}

The proof proceeds in three steps which exactly parallel the analysis for $d=4$, as follows.

1. A single $R G$ step. We estimate the map representing a single $\mathrm{RG}$ step using the results of [16, Section 1.8]. The domain for $V$ is given by (B.9), and $\tilde{g}$ in [16] becomes instead $\tilde{a}$.

2. Global bulk $R G$ flow. The construction of the $\left(m^{2}, a_{0}\right)$-dependent critical initial condition $\left(g_{0}^{c}, \nu_{0}^{c}, z_{0}^{c}\right)$ for the global bulk RG flow (without observables) in Theorem 2.1 is achieved using an adaptation of [7, Theorem 1.4]. The adaptation of [7] is notational only, to take into account that there now are two relevant variables $g, \nu$ rather than just $\nu$.

3. Global observable flow. The bulk flow is independent of the flow of the observable coupling constants $(\lambda, q)$. Unlike the construction of the critical initial condition in Step 2, which involves what is essentially a delicate implicit function theorem (couched in the context of local existence theory for ODEs in [7]), the observable flow is simply solved forward recursively from the initial condition. This requires a relatively straightforward induction argument, which can be carried out just as in [4, Section 4] (or as in [35]). In Theorem 2.2, we have isolated the estimates produced by the induction argument. The part of that argument involving the coupling constants is given in Section 4.2 to illustrate the calculations that lead to our main result. 


\section{Acknowledgements}

The work of ML and GS was supported in part by NSERC of Canada. The work of GS was partially supported by a grant from the Simons Foundation. The authors would like to thank the Isaac Newton Institute for Mathematical Sciences for support and hospitality during the programme "Scaling limits, rough paths, quantum field theory" when work on this paper was undertaken; this work was supported by EPSRC Grant Number EP/R014604/1. GS is grateful to Takashi Kumagai and Ryoki Fukushima for support and hospitality at the Research Institute for Mathematical Sciences, Kyoto University, where part of this work was carried out. We thank David Brydges for helpful comments on a preliminary version of the paper.

\section{References}

[1] D. Arovas. Lecture notes on thermodynamics and statistical mechanics. In preparation. https://courses.physics. ucsd.edu/2017/Spring/physics210a/LECTURES/BO0K_STATMECH.pdf , (2018).

[2] R. Bauerschmidt. A simple method for finite range decomposition of quadratic forms and Gaussian fields. Probab. Theory Related Fields, 157:817-845, (2013).

[3] R. Bauerschmidt, D.C. Brydges, and G. Slade. Scaling limits and critical behaviour of the 4-dimensional $n$-component $|\varphi|^{4}$ spin model. J. Stat. Phys, 157:692-742, (2014).

[4] R. Bauerschmidt, D.C. Brydges, and G. Slade. Critical two-point function of the 4-dimensional weakly self-avoiding walk. Commun. Math. Phys., 338:169-193, (2015).

[5] R. Bauerschmidt, D.C. Brydges, and G. Slade. Logarithmic correction for the susceptibility of the 4-dimensional weakly self-avoiding walk: a renormalisation group analysis. Commun. Math. Phys., 337:817-877, (2015).

[6] R. Bauerschmidt, D.C. Brydges, and G. Slade. A renormalisation group method. III. Perturbative analysis. J. Stat. Phys, 159:492-529, (2015).

[7] R. Bauerschmidt, D.C. Brydges, and G. Slade. Structural stability of a dynamical system near a non-hyperbolic fixed point. Ann. Henri Poincaré, 16:1033-1065, (2015).

[8] R. Bauerschmidt, D.C. Brydges, and G. Slade. Introduction to a Renormalisation Group Method. Springer, Singapore, (2019). Lecture Notes in Mathematics Vol. 2242.

[9] R. Bauerschmidt and G. Slade. Mean-field tricritical polymers. https://arxiv.org/abs/1911.00395, (2019).

[10] R. Bauerschmidt, G. Slade, and B.C. Wallace. Four-dimensional weakly self-avoiding walk with contact self-attraction. J. Stat. Phys, 167:317-350, (2017).

[11] N.R. Beaton, A.J. Guttmann, and I. Jensen. Two-dimensional interacting self-avoiding walks: new estimates for critical temperatures and exponents. https://arxiv.org/abs/1911.05852, (2019). 
[12] D.C. Brydges, G. Guadagni, and P.K. Mitter. Finite range decomposition of Gaussian processes. J. Stat. Phys., 115:415-449, (2004).

[13] D.C. Brydges and G. Slade. A renormalisation group method. I. Gaussian integration and normed algebras. J. Stat. Phys, 159:421-460, (2015).

[14] D.C. Brydges and G. Slade. A renormalisation group method. II. Approximation by local polynomials. J. Stat. Phys, 159:461-491, (2015).

[15] D.C. Brydges and G. Slade. A renormalisation group method. IV. Stability analysis. J. Stat. Phys, 159:530-588, (2015).

[16] D.C. Brydges and G. Slade. A renormalisation group method. V. A single renormalisation group step. J. Stat. Phys, 159:589-667, (2015).

[17] B. Duplantier. Tricritical polymer chains in or below three dimensions. Europhys. Lett., 1:491-498, (1986).

[18] B. Duplantier. Geometry of polymer chains near the theta-point and dimensional regularization. J. Chem. Phys., 86:4233-4244, (1987).

[19] J. Feldman, J. Magnen, V. Rivasseau, and R. Sénéor. Construction and Borel summability of infrared $\Phi_{4}^{4}$ by a phase space expansion. Commun. Math. Phys., 109:437-480, (1987).

[20] K. Gawȩdzki and A. Kupiainen. Massless lattice $\varphi_{4}^{4}$ theory: Rigorous control of a renormalizable asymptotically free model. Commun. Math. Phys., 99:199-252, (1985).

[21] S.E. Golowich and J.Z. Imbrie. The broken supersymmetry phase of a self-avoiding random walk. Commun. Math. Phys., 168:265-319, (1995).

[22] A. Hammond and T. Helmuth. Self-attracting self-avoiding walk. Probab. Theory Related Fields, 175:677-719, (2019).

[23] T. Hara and H. Tasaki. A rigorous control of logarithmic corrections in four dimensional $\varphi^{4}$ spin systems. II. Critical behaviour of susceptibility and correlation length. J. Stat. Phys., 47:99-121, (1987).

[24] R. van der Hofstad, A. Klenke, and W. König. The critical attractive random polymer in dimension one. J. Stat. Phys., 106:477-520, (2002).

[25] R. van der Hofstad and W. König. A survey of one-dimensional random polymers. J. Stat. Phys., 103:915-944, (2001).

[26] F. den Hollander. Random Polymers. Springer, Berlin, (2009). Lecture Notes in Mathematics Vol. 1974. Ecole d'Eté de Probabilités de Saint-Flour XXXVII-2007.

[27] C. Itzykson and J-M. Drouffe. Statistical Field Theory, volume II. Cambridge University Press, Cambridge, (1989).

[28] G.F. Lawler. Intersections of Random Walks. Birkhäuser, Boston, (1991). 
[29] M. Lohmann. Critical exponent for the magnetization of the weakly coupled $\phi_{4}^{4}$ model. https://arxiv.org/abs/1810.13138, (2018).

[30] M. Lohmann, G. Slade, and B.C. Wallace. Critical two-point function for long-range $O(n)$ models below the upper critical dimension. J. Stat. Phys., 169:1132-1161, (2017).

[31] P.K. Mitter and B. Scoppola. Renormalization group approach to interacting polymerised manifolds. Commun. Math. Phys., 209:207-261, (2000).

[32] V.F. Müller and J. Schiemann. Infrared asymptotic freedom of a hierarchical $\phi_{3}^{6}$ lattice theory. J. Stat. Phys., 43:123-142, (1986).

[33] N. Pétrélis and N. Torri. Collapse transition of the interacting prudent walk. Ann. Inst. Henri Poincaré Comb. Phys. Interact., 5:387-435, (2018).

[34] G. Slade. Critical exponents for long-range $O(n)$ models below the upper critical dimension. Commun. Math. Phys., 358:343-436, (2018).

[35] G. Slade and A. Tomberg. Critical correlation functions for the 4-dimensional weakly selfavoiding walk and $n$-component $|\varphi|^{4}$ model. Commun. Math. Phys., 342:675-737, (2016).

[36] M.J. Stephen, E. Abrahams, and J.P. Straley. Logarithmic corrections to the mean-field theory of tricritical points. Phys. Rev. B, 12:256-262, (1975).

[37] D. Ueltschi. A self-avoiding walk with attractive interactions. Probab. Theory Related Fields, 124:189-203, (2002).

[38] F.J. Wegner and E.K. Riedel. Logarithmic corrections to the molecular-field behavior of critical and tricritical systems. Phys. Rev. B, 7:248-256, (1973). 